Published in final edited form as:

Nat Genet. 2019 November ; 51(11): 1596-1606. doi:10.1038/s41588-019-0513-9.

\title{
Loss of ZnT8 function protects against diabetes by enhanced insulin secretion
}

\begin{abstract}
Om Prakash Dwivedi ${ }^{\# 1}$, Mikko Lehtovirta ${ }^{\# 1}$, Benoit Hastoy ${ }^{\# 2}$, Vikash Chandra ${ }^{3}$, Nicole A. J. Krentz $^{4}$, Sandra Kleiner ${ }^{5}$, Deepak Jain ${ }^{6}$, Ann-Marie Richard ${ }^{7}$, Fernando Abaitua ${ }^{4}$, Nicola L. Beer $^{2}$, Antje Grotz ${ }^{2}$, Rashmi B. Prasad ${ }^{8}$, Ola Hansson ${ }^{1,8}$, Emma Ahlqvist ${ }^{8}$, Ulrika Krus ${ }^{8}$, Isabella Artner ${ }^{8}$, Anu Suoranta ${ }^{1}$, Daniel Gomez ${ }^{5}$, Aris Baras ${ }^{5}$, Benoite Champon ${ }^{4}$, Anthony J Payne $^{4}$, Daniela Moralli ${ }^{4}$, Soren K. Thomsen ${ }^{2}$, Philipp Kramer ${ }^{4}$, Ioannis Spiliotis ${ }^{2}$, Reshma Ramracheya ${ }^{2}$, Pauline Chabosseau ${ }^{9}$, Andria Theodoulou ${ }^{9}$, Rebecca Cheung ${ }^{9}$, Martijn van de Bunt ${ }^{2,4}$, Jason Flannick ${ }^{10,11}$, Maddalena Trombetta ${ }^{12}$, Enzo Bonora ${ }^{12}$, Claes B. Wolheim ${ }^{8}$, Leena Sarelin ${ }^{13}$, Riccardo C. Bonadonna ${ }^{14}$, Patrik Rorsman ${ }^{2}$, Benjamin Davies $^{4}$, Julia Brosnan ${ }^{7}$, Mark I. McCarthy ${ }^{2,4,15}$, Timo Otonkoski ${ }^{3}$, Jens O. Lagerstedt ${ }^{6}$, Guy

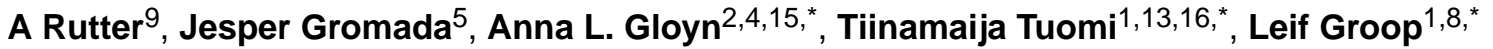

${ }^{1}$ Institute for Molecular Medicine Finland (FIMM), Helsinki University, Helsinki, Finland ${ }^{2}$ Oxford Centre for Diabetes Endocrinology \& Metabolism, University of Oxford, UK ${ }^{3}$ Stem Cells and Metabolism Research Program and Biomedicum Stem Cell Centre, Faculty of Medicine, University of Helsinki, Finland ${ }^{4}$ Wellcome Centre for Human Genetics, University of Oxford, UK ${ }^{5}$ Regeneron Pharmaceuticals, Tarrytown, New York, USA ${ }^{6}$ Department of Experimental Medical
\end{abstract}

Users may view, print, copy, and download text and data-mine the content in such documents, for the purposes of academic research, subject always to the full Conditions of use:http://www.nature.com/authors/editorial_policies/license.html\#terms

Correspondence: Leif Groop, Institute for Molecular Medicine Finland (FIMM), Helsinki University. Leif. Groop@ helsinki.fi and/or leif.Groop@med.lu.se.

These authors jointly supervised this study

Reporting Summary.

Further information on research design is available in the Nature Research Reporting Summary linked to this article.

Data Availability

Individual level data for the human study can only be obtained via the Biobank of The Institute of Health and Welfare in Finland (https://thl.fi/en/web/thl-biobank). Next-generation sequencing data have been deposited in the SRA data base (PRJNA563975) and its processed counts data could be found in the Supplementary Dataset 1. The individual processed data from cell lines (Fig. 4 and 5 ), mice studies (Fig. 6) and human islet work (Fig. 7) are available in the Source Data files. Additional data supporting the findings of this study are available on request from the corresponding author.

Author Contributions

M.L., L.S., T.T. and L.G. conducted the human study; E.A., O.H., A.B., O.P.D. and J.F. analyzed the genotype data ; M.L., O.P.D., M.T., E.B., R.C.B, T.T. and L.G. analyzed the human data; B.H., A.G, N.L.B., S.K.T., M.vD.B., V.C., O.P.D., T.O. and A.L.G. characterized the Human beta-cell model; N.A.J.K., F.A., N.L.B., B.C., D.M., P.K., B.D., O.P.D., A.S., M.I.M. and A.L.G. characterized the human IPS cell derived model; U.K., R.B.P., O.P.D., B.H., A.J.P., I.S., R.R., I.A., P.R., M.I.M. and A.L.G. characterized the human islets; S.K., D.G. and J.G. characterized the Slc30a8 p.Arg138* mice; D.J., J.O.L., P.C., A.T., R.C., A-M.R., J.B. and G.A.R. characterized the rat insulinoma cell-line; M.I.M., A.L.G., T.T. and L.G. supervised the project; O.P.D., M.L., B.H., N.A.J.K., S.K., P.R., C.B.W., A.L.G., T.T., and L.G. wrote the manuscript; all authors revised the manuscript.

Competing interests

L.G. has received research funding from Pfizer Inc, Regeneron Pharmaceuticals, Eli Lilly and Astra Zeneca. N.L.B. and M.vD.B are now employees of Novo Nordisk, although all experimental work was carried out under employment at the University of Oxford. ALG has received honoraria from Novo Nordisk and Merck. MIM serves on advisory panels for Pfizer, Novo Nordisk, Zoe Global; has received honoraria from Pfizer, Novo Nordisk and Eli Lilly; has stock options in Zoe Global; has received research funding from Abbvie, Astra Zeneca, Boehringer Ingelheim, Eli Lilly, Janssen, Merck, Novo Nordisk, Pfizer, Roche, Sanofi Aventis, Servier, Takeda. GAR is a consultant for Sun Pharma and has received grant funding from Servier. J.O.L. has received research funding from Pfizer Inc and Novo Nordisk A/S. 
Science, Lund University, 221 84, Lund, Sweden ${ }^{7}$ Pfizer Inc, Cambridge, MA, United States of America ${ }^{8}$ Lund University Diabetes Centre, Department of Clinical Sciences, Lund University, Skåne University Hospital, SE-20502 Malmö, Sweden ${ }^{9}$ Section of Cell Biology, Department of Medicine, Imperial College London, Imperial Centre for Translational and Experimental Medicine, Hammersmith, Hospital, Du Cane Road, London, W12 0NN, UK ${ }^{10}$ Department of Molecular Biology, Massachusetts General Hospital, Boston, Massachusetts, USA ${ }^{11}$ Program in Medical and Population Genetics, Broad Institute, Cambridge, Massachusetts, USA ${ }^{12}$ Department of Medicine, University of Verona and Azienda Ospedaliera Universitaria Integrata of Verona, Verona, Italy ${ }^{13}$ Folkhälsan Research Center, Helsinki, Finland ${ }^{14}$ Department of Medicine and Surgery, University of Parma School of Medicine and Azienda Ospedaliera Universitaria of Parma, Italy ${ }^{15}$ Oxford NIHR Biomedical Research Centre, Churchill Hospital, Oxford, UK ${ }^{16}$ Abdominal Center, Endocrinology, Helsinki University Central Hospital; Research Program for Clinical and Molecular Metabolism, University of Helsinki, Helsinki, Finland

\# These authors contributed equally to this work.

\section{Abstract}

A rare loss-of-function allele p.Arg138* in SLC30A8 encoding the zinc transporter 8 (ZnT8), enriched in Western Finland, protects against type 2 diabetes (T2D). We recruited relatives of the identified carriers and showed that protection was associated with better insulin secretion due to enhanced glucose responsiveness and proinsulin conversion, particularly when compared with individuals matched for the genotype of a common T2D-risk allele in SLC3OA8, p.Arg325. In genome-edited human iPSC-derived $\beta$-like cells, we establish that the p.Arg $138^{*}$ allele results in reduced SLC30A8 expression due to haploinsufficiency. In human $\beta$-cells, loss of SLC30A8 leads to increased glucose responsiveness and reduced $\mathrm{K}_{\mathrm{ATP}}$ channel function similar to isolated islets from carriers of the T2D-protective allele p.Trp325. These data position ZnT8 as an appealing target for treatment aimed at maintaining insulin secretion capacity in T2D.

Zinc transporters (ZNT) regulate the passage of zinc across biological membranes out of the cytosol, while Zrt/Irt-like proteins (ZIP) transport zinc into the cytosol ${ }^{1}$. ZnT8, encoded by $S L C 30 A 8$, is highly expressed in the membrane of insulin granules within pancreatic $\beta$ cells, where it transports zinc ions for crystallization and storage of insulin ${ }^{2}$. We previously described a loss-of-Function (LoF) allele p.Arg138* (rs200185429, c.412C >T) in the SLC30A8 gene that conferred 53\% protection against $\mathrm{T}_{2} \mathrm{D}^{3}$. This allele was extremely rare $(0.02 \%)$ in most European countries but more common $(>0.2 \%)$ in Western Finland ${ }^{3}$. We also reported a protective frameshift allele p.Lys 34 Serfs $50 *$ conferring $83 \%$ protection against T2D in Iceland ${ }^{3}$. Further, the $S L C 30 A 8$ gene harbors a common variant (rs13266634, c.973C >T) p.Trp325Arg in the C-terminal domain ${ }^{4}$. Whilst the major p.Arg325 allele ( $>70 \%$ of the population) confers increased risk for T2D, the minor p.Trp325 allele is protective ${ }^{5}$.

The mechanisms by which modulation of ZnT8 activity protects against T2D are largely unknown. Several attempts have been made to study loss of Slc30a8 function in rodent models, but the results have been inconclusive; global knock-out of Slc30a8 led to either 
glucose intolerance or had no effect in mice ${ }^{6,7,8}$, whilst over-expression improved glucose tolerance without effect on insulin secretion ${ }^{9}$. A mouse model harboring the equivalent of the human p.Arg138* allele lacked any detectable ZnT8 protein but showed no effect on glucose tolerance ${ }^{10}$. These rodent studies present a complex picture that may not recapitulate the T2D protective effects of $S L C 30 A 8 \mathrm{LoF}$ alleles in humans. We therefore performed detailed metabolic studies in human carriers heterozygous for the LoF allele (p.Arg138*) recruited on the basis of their genotype, performed comprehensive functional studies in human $\beta$-cell models, and compared these with the mouse model carrying the human p.Arg $138 *-$ Slc30a8 allele.

\section{Results}

\section{Recruitment by genotype}

Given the enrichment of the p.Arg138*-SLC30A8 allele in Western Finland, we genotyped $>14,000$ individuals from the Botnia Study ${ }^{11}$ for the SLC30A8 p.Arg 138* and the common p.Trp325Arg variants, and identified 71 p.Arg 138*-SLC30A8 carriers (all heterozygotes; 55 non-diabetic individuals, Fig. 1). We then recruited family members of known p.Arg $138^{*}$ carriers to identify additional p.Arg $138^{*}$ carriers to perform a detailed metabolic study (190 minutes test meal) in carriers and non-carrier relatives. Of the 79 p.Arg 138* carriers (65 novel, 14 previously identified) and 103 non-carrier relatives from $>21$ families (Extended Data Fig. 1), 54 and 47, respectively, participated in a test meal and 31 and 13 participated in an oral glucose tolerance test (OGTT) during a separate second visit (Fig. 1, Supplementary Table 1 and 2). We also had data from previously performed OGTTs within the Botnia Study for 8,436 non-diabetic individuals (55 p.Arg 138* carriers, Fig. 1, Supplementary Table 2 and 3). Of the 136 p.Arg $138^{*}$ allele carriers, none were homozygous for the protective common variant, p.Trp325, and p.Arg 138* segregated with p.Arg325 in all families (Extended Data Fig. 1). Thus, we present the data in three different ways: 1) p.Arg138* vs. all p.Arg138Arg, 2) p.Arg138* vs. p.Arg138Arg having at least one p.Arg325 allele (p.Trp325Arg or p.Arg325Arg), and 3) p.Arg325 (p.Trp325Arg or p.Arg325Arg) vs. p.Trp325Trp on a background of p.Arg138Arg.

Replicating our previous findings ${ }^{3}$, carriers of $\mathrm{p} . \mathrm{Arg} 138 *$ had a reduced risk of T2D (OR $=$ $0.40, p=0.003)$ when analyzing 4,564 T2D (13 p.Arg138* carriers) and 8,183 non-diabetic (55 p.Arg $138 *$ carriers) individuals. Additionally, non-diabetic p.Arg 138* carriers had lower fasting glucose concentrations than p.Arg138Arg individuals (Supplementary Table 4 and 5). There were no significant differences in plasma zinc concentrations measured during test meal or OGTT between the groups (data not shown).

Comparison of p.Arg138* vs. p.Arg138Arg-The p.Arg138* carriers had lower blood glucose levels during the test meal especially during the first 40 minutes (area under curve, $p$ $=0.02$ ) and showed a higher corrected insulin response (CIR) (at 20 minutes, $p=0.046$ ) than non-carriers (Supplementary Tables 6 and Fig. 2a-c). Similarly, in the larger populationbased OGTT cohort, carriers had higher insulin response during OGTT (Fig 3a-c, left panel), especially with respect to the early incremental insulin response $(p=0.008)$ and the insulin/glucose ratio (at 30 minutes, $p=0.002$, Supplementary Table 4 ). The higher insulin 
secretory response during OGTT was consistent across different subsets with OGTT data (meta-analysis: CIR, $\mathrm{P}=0.002$; incremental insulin, $\mathrm{p}=2.4 \times 10^{-4} ; 30$ minutes insulin, $\mathrm{p}=$ $\left.3.8 \times 10^{-4}\right)($ Supplementary Table 5). Of note, the p.Arg138* carriers had significantly lower proinsulin/C-peptide (20 minutes: $\mathrm{p}=0.041 ; 40$ minutes: $\mathrm{p}=0.043$ ) and proinsulin/insulin (20 minutes: $\mathrm{p}=0.006$ ) ratios during the test meal suggesting effects on proinsulin conversion (Fig. 2d-e). No differences were seen in glucagon, GLP-1 or free fatty acid concentrations during the test meal (Extended Data Fig. 2c-e). Neither model-based insulin clearance index nor the ratio of insulin and C-peptide areas under the curve during the test meal differed between p.Arg138* and p.Arg138Arg (Extended Data Fig. 2f-g).

Comparison of p.Arg138* vs. p.Arg138Arg-p.Arg325-The above differences were magnified when we restricted the p.Arg138Arg group to carriers of the common risk variant p.Arg325 (middle panel of Fig. 2). The early phase (0-40 minutes) insulin ( $p=0.026)$, insulin/glucose ratio $(\mathrm{p}=0.004)$ and CIR ( $\mathrm{p}=0.004 ; 20$ minutes) were all greater in p.Arg138* carriers vs. p.Arg138Arg on a background of p.Arg325 (Supplementary Table 6). Both the proinsulin/C-peptide (20 minutes: $\mathrm{p}=0.027,40$ minutes: $\mathrm{P}=0.044$ ) and proinsulin/insulin ratios (20 minutes: $\mathrm{P}=0.003)$ were reduced in p.Arg138* carriers (middle panel of Fig. 2d-e).

Comparison of p.Trp325Trp vs. p.Arg325-The effect of p.Trp325Trp genotype on glucose and insulin response mimicked the effects of p.Arg138* with pronounced early (20 minutes) insulin $(\mathrm{p}=0.032)$ and $\mathrm{C}$-peptide $(\mathrm{p}=0.030)$ responses during the test meal (right panel of Fig. 2b-c and Extended Data Fig. 2a), as well as increased insulin secretion (30 minutes insulin: $\mathrm{p}=0.003,30$ minutes insulin/glucose: $\mathrm{p}=0.002$, incremental insulin: $\mathrm{p}=$ $0.005)$, lower fasting and 120 minute proinsulin $(\mathrm{p}=0.006$ and $\mathrm{p}=0.039$, respectively) concentration during OGTT in p.Trp325 carriers (Supplementary Table 4, right panel of Fig. 3b-c). Moreover, $p$. Trp325Trp carriers showed a pronounced increased $(p=0.003)$ early incremental insulin secretion during the intravenous glucose tolerance test (IVGTT) (Extended Data Fig. 3a-b and Supplementary Table 4). In patients with newly diagnosed T2D (Supplementary Table 7), the p.Trp325Trp carriers showed a trend $(\mathrm{p}=0.12)$ towards enhanced $\beta$-cell sensitivity to glucose during the OGTT (Extended Data Fig. 3c).

Taken together, all human in vivo data on p.Arg138* show enhanced glucose-stimulated insulin secretion combined with enhanced proinsulin conversion as a potential explanation for T2D protection. The common allele p.Trp325 is also associated with protection from T2D and the metabolic effects are similar but more modest than those of on p.Arg 138*, suggesting it also might reduce ZnT8 function.

\section{SLC30A8 p.Arg138* and p.Lys34Serfs50* in human iPSCs}

The majority of nonsense SLC30A8 alleles (including p.Arg138* and p.Lys34Serfs50*) which protect against T2D are located in the first four exons of the eight-exon canonical islet SLC30A8 transcript (ENST00000456015) and are predicted to undergo nonsense mediated decay (NMD), a cell surveillance pathway that reduces errors in gene expression by eliminating mRNA transcripts with premature stop codons. To confirm that nonsense $S L C 30 A 8$ alleles lead to haploinsufficiency through NMD, we used CRISPR-Cas9 to 
introduce two protective alleles, p.Arg138* and p.Lys34Serfs50*, into the SLC30A8 locus of the SB Ad3.1 human induced pluripotent stem cell (iPSC) line (Extended Data Fig. 4a). Two heterozygous hiPSC lines for the SLC30A8-p.Arg138* allele (clone B1 and A3) and two homozygous hiPSC lines for SLC30A8-p.Lys34Serfs50* (clone B3 and D3) were generated and compared with an unedited wildtype and a CRISPR-Sham hiPSC line. All hiPSC lines passed quality control checks including karyotyping and pluripotency (Extended Data Fig. 4b-c).

We subjected our SLC30A8-edited iPSCs to a previously published in vitro endocrine pancreas differentiation protocol ${ }^{12}$ (Extended Data Fig. 4). At the end of the seven stage protocol, SLC3OA8 expression was significantly reduced in cells heterozygous for the p.Arg 138* allele (clone A3 0.06 $\pm 0.03, \mathrm{p}<0.0001$; clone B1 $0.04 \pm 0.01, \mathrm{p}<0.0001$ ) or homozygous for the p.Lys34Serfs50* (clone B3 0.04 \pm 0.01 , p $<0.0001$; clone D3 0.04 \pm 0.01 , $\mathrm{p}<0.0001)$ compared to unedited control cells $(1.02 \pm 0.13)$ (Fig. 4a). In addition, ZnT8 protein was absent in homozygous p.Lys34Serfs50* hiPSC-derived beta-like cells compared to wildtype controls, but was also undetectable in differentiated heterozygous p.Arg138* clones (Fig. 4b-c), suggesting an impact on differentiation. Indeed, the number of INS- and SLC30A8-transcript expressing cells were reduced in clones with premature stop codons in SLC30A8 (Extended Data Fig. 4d-g), indicating a reduced formation of beta-like cells. To disentangle effects on differentiation from those on expression, allele-specific SLC3OA8 expression was quantified by digital droplet $\mathrm{PCR}^{13}$ in the heterozygous p.Arg138* lines. Of note, p.Arg 138* allele-specific SLC30A8 expression was reduced compared to the WT allele (clone A3: $24.3 \pm 3.1 \%, \mathrm{p}<0.0001$; clone B1: 22.2 $\pm 1.7 \%, \mathrm{p}<0.0001$ ) (Fig. $4 \mathrm{~d}$ ). The reduced expression of the mutant SLC30A8 allele (clone A3: $18.2 \pm 1.8 \%$; clone B1: $18.6 \%$;) compared to the wildtype allele $(81.7 \pm 1.3 \%)$ was confirmed $(\mathrm{p}<0.0001)$ by targeted SLC3OA8 mRNA sequencing (Fig. $4 \mathrm{e}$ and Extended Data Fig. 5c). Although not statistically significant, inhibition of NMD by cycloheximide showed a trend towards a greater rescue of the p.Arg $138 *$ transcript compared to the p.Arg138Arg transcript (clone A3: $230 \pm 61 \%$ vs. $150 \pm 33 \%, \mathrm{p}=0.28$ and clone B1:198 $\pm 45 \%$ vs. $152 \pm 26 \%$ p=0.39, Fig. 4f-g). Taken together, these data show that the protective p.Arg $138 *-S L C 30 A 8$ allele undergoes NMD, resulting in haploinsufficiency for SLC3OA8.

\section{Impact of SLC30A8 loss in a human $\beta$-cell line}

Since human in vivo studies provided strong evidence for a role of the p.Arg138* on insulin secretion and proinsulin processing, we studied the impact of SLC3OA8 loss using siRNA mediated knockdown on both phenotypes in a well characterized human $\beta$-cell model EndoC- $\beta \mathrm{H} 1{ }^{14}$. By siRNA, we achieved a 30-65\% decrease in SLC30A8 mRNA levels (Fig. $5 a ; p=0.003$, Extended Data Fig. 7a; $p=5.93 \times 10^{-22}$ ) which resulted in reduced ZnT8 protein levels (Fig. 5b, Extended Data Fig. 6a) associated with a reduction in intracellular zinc content (34\%, p = 0.002, Fig. 5c). Assessment of dense core granule zinc content in SLC30A8-silenced EndoC- $\beta \mathrm{H} 1$ cells (Extended Data Fig. 6a), through the use of the cell surface attached fluorescent zinc probe ZIMIR ${ }^{15}$ (Extended Data Fig. 6b-c) showed a tendency ( $\mathrm{p}=0.10$, Extended Data Fig. $6 \mathrm{~d}$ ), for reduced $\mathrm{Zn}^{2+}$ release, consistent with the loss of zinc from this compartment, and also with previous findings in islets from Slc30a8 null mice ${ }^{15}$. 
Knockdown of $S L C 30 A 8$ had no significant effect on stimulated insulin secretion neither in response to $20 \mathrm{mM}$ glucose nor to the sulphonylurea tolbutamide (which closes $\mathrm{K}_{\mathrm{ATP}}$ channels, Fig. 5d). However, basal insulin secretion was significantly increased in siSLC30A8 transfected cells $(\mathrm{p}=0.048)$ which reduced marginally the glucose stimulation index $(\mathrm{p}=0.26$, Fig. $5 \mathrm{e})$. In addition, the inhibitory effect of diazoxide, a $\mathrm{K}_{\mathrm{ATP}}$ channel opener, on glucose-stimulated insulin secretion, was significantly reduced ( $\mathrm{p}=8 \times 10^{-3}$, Fig. 5d). There was no effect of $S L C 30 A 8$ knockdown on insulin content (Fig. 5f). We then measured the resting membrane conductance $\left(\mathrm{G}_{\mathrm{m}}\right)$ in siSLC3OA8 transfected cells incubated with $100 \mu \mathrm{M}$ diazoxide (to activate $\mathrm{K}_{\mathrm{ATP}}$ channels). In control cells, $\mathrm{G}_{\mathrm{m}}$ was in agreement with previous reports ${ }^{16}$, while $S L C 30 A 8$ knockdown reduced $\mathrm{G}_{\mathrm{m}}$ by $65 \%(\mathrm{p}=0.002$, Fig. $5 \mathrm{~g}$ ) without altering cell size (Fig. $5 \mathrm{~h}$ ). Indeed, reduced $\mathrm{K}_{\mathrm{ATP}}$ channel activity is consistent with the reduced expression observed of the genes encoding the $\mathrm{K}_{\mathrm{ATP}}$ channel subunits, SUR1 ( $A B C C 8)$ and Kir6.2 (KCNJ11) (P = 0.04 and 0.06 respectively, Fig. 5i). In addition, insulin secretion elicited by the combination of elevated $50 \mathrm{mM}$ extracellular $\mathrm{K}^{+}\left(\left[\mathrm{K}^{+}\right]_{\mathrm{o}}\right)$ to depolarize the cells and open voltage-gated $\mathrm{Ca}^{2+}$ channels and $16.7 \mathrm{mM}$ glucose was significantly higher after $S L C 30 A 8$ knockdown $(\mathrm{p}=0.002$, Fig. $5 \mathrm{j})$. The proinsulin-insulin ratios (both total content; $p=0.007$, secreted hormones; $p=0.004$ ) and proinsulin concentrations (total content; $\mathrm{p}=0.011$, secreted in media; $\mathrm{p}=0.017$ ) were decreased in siSLC30A8 transfected cells, but no differences in either protein or mRNA levels of genes (PCSK1, CPE and PCSK2) involved in proinsulin processing in SLC3OA8 KD cells was observed (Fig. 5m-q, Extended Fig. 7a). However, we did observe increased AKT phosphorylation (pAKT-473) and improved cell survival under ER stress $(\mathrm{p}=0.016$ and 0.032 respectively, Fig. 5r-t) in SLC30A8 KD cells.

We next examined the effects of $S L C 30 A 8$ knockdown on gene expression in EndoC- $\beta \mathrm{H} 1$ cells by mRNA sequencing (RNA-seq) of siSLC3OA8 treated and siScramble cells ( $\mathrm{n}=8 \mathrm{vs}$. 8 , see Supplementary Note). We observed a total of 674 significantly differently expressed genes among 12,956 protein coding genes that passed the quality control filters (Extended Data Fig. 7 and Supplementary Dataset 1). RNA-seq confirmed the reduction in KCNJ11 and $A B C C 8$ expression in SLC3OA $8 \mathrm{KD}$ cells $\left(\mathrm{p}=2.6 \times 10^{-18}\right.$ and $\mathrm{p}=2.04 \times 10^{-9}$ respectively, Extended Data Fig. 7c) as seen earlier by qPCR (Fig. 5i). In addition, further genes involved in the regulation of $\beta$-cell excitability/exocytosis, including $C A C N A 1 C$, were up-regulated in KD cells (Extended Data Fig. 7c). Moreover, expression of genes associated with $\beta$-cell maturation and development were also influenced by $S L C 30 A 8$ knockdown with decreased expression of $N K X 6.1$ and $P D X 1$ and increased expression of SOX4, SOX6 and SOX11 (Extended Data Fig. 7b). A pathway enrichment analysis of differentially expressed genes showed enrichment of genes involved in the WNT signaling and insulin secretion pathways (Extended Data Fig. 7 and Supplementary Dataset 1). A global gene set enrichment analysis (GSEA) of all expressed genes $(\mathrm{N}=12,956)$ using a gene ontology database showed enrichment of genes involved in positive regulation of TOR signaling (Extended Data Fig. 7) in KD cells. Collectively, these data demonstrate a link between $S L C 30 A 8$ expression and transcriptional networks involved in cell development, cell fate and plasma membrane polarization. 


\section{Metabolic phenotype of SIc30a8 p.Arg138* mice}

Since neither global nor tissue-specific SIc30a8 knockout mouse models have recapitulated the human phenotype in carriers of the SLC3OA8 p.Arg138* allele, we attempted to overcome this problem by using a mouse model carrying the human Slc30a8 p.Arg138* allele ${ }^{10}$. On a standard chow diet there was no evidence of enhanced insulin secretion ${ }^{10}$ but when subjected to a high fat diet (HFD) the mice showed a significant increase in insulin secretion $(p=0.0016$, Fig. 6i), and similar changes in proinsulin/insulin $(p=0.0004)$ and proinsulin/C-peptide ratios $(\mathrm{p}=0.0001$ ) as seen in human carriers (Fig. $6 \mathrm{f}-\mathrm{g}$ ). No significant changes were seen in insulin clearance, glucose or insulin tolerance (Fig. 6h, j, k).

\section{Impact of p.Arg138* on cytosolic zinc in INS-1 cells}

Although we found no evidence for the presence of a truncated protein in the human p.Arg138* $\beta$-cell model (Fig. 4) as also reported for the p.Arg138* mice ${ }^{10}$, we still explored the possibility that a truncated protein could result from mRNA evading NMD. Transient overexpression of tagged ZnT8-p.Arg138* fusion protein in a rat insulinoma cell line, INS-1e, showed distinct punctate distribution patterns, consistent with localization of the truncated ZnT8 protein to secretory granules, as previously observed with the full length protein ${ }^{17}$ (Extended Data Fig. 8a-c). Additionally, Western blot showed stable expression of truncated ZnT8 in native INS1e cells (Extended Data Fig. 8d).

To investigate the effects of a truncated $\mathrm{ZnT} 8$ protein on cytosolic free $\mathrm{Zn}^{2+}$, we used a genetically-encoded $\mathrm{Zn}^{2+}$ sensor eCALWY- ${ }^{18}$. Overexpression of the truncated protein (p.Arg138*, Extended Data Fig. 8e-f) had no impact on cytosolic free $\mathrm{Zn}^{2+}$ when expressed in INS-1(832/13) cells (Extended Data Fig. 8g).

\section{Influence of SLC30A8 p.Trp325Arg in primary human islets}

Although we were unable to study the effects of the rare T2D-protective alleles in primary human islets, we were able to assess the impact of the p.Trp325Arg genotype on in vitro insulin and glucagon secretion. Islets obtained from cadaveric p.Trp325 carriers secreted more insulin $(\mathrm{p}=0.0153)$ than $\mathrm{p} . \operatorname{Arg} 325 \mathrm{Arg}$ carriers when stimulated with high glucose $(16.7 \mathrm{mM})$ and depolarizing $\left[\mathrm{K}^{+}\right]_{\mathrm{o}}(70 \mathrm{mM})$, in line with findings of $S L C 30 A 8$ knockdown in EndoC- $\beta \mathrm{H} 1$ (Fig. 7a-b). Interestingly, a trend towards increased glucose responsiveness was already observed at submaximal glucose stimulation $(6 \mathrm{mM})$ (Fig. 7c). Increasing glucose from $1 \mathrm{mM}$ to $6 \mathrm{mM}$ stimulated insulin secretion $2.2(\mathrm{p}=0.031)$ and $2.7(\mathrm{p}=0.012)$ fold in p.Arg325 and p.Trp325 carriers respectively, with no effect on insulin content (Fig. $7 \mathrm{c}-\mathrm{d})$.

As $S L C 30 A 8$ is highly expressed in human alpha cells ${ }^{1}$, we also measured glucagon secretion from the same islets (Fig. 7e-f). In islets from p.Arg325Arg donors, $6 \mathrm{mM}$ glucose inhibited glucagon secretion by $\sim 50 \%$ compared to $1 \mathrm{mM}$ glucose. In islets from p.Trp325Arg donors, glucagon secretion at $1 \mathrm{mM}$ glucose was reduced by $50 \%(\mathrm{p}=0.033)$ compared to p.Arg325Arg donors with no effect on glucagon content (Fig. 7e-f).

Finally we explored co-expression of $S L C 30 A 8$ with relevant candidate genes (INS, GCG, encoding proinsulin processing and $\mathrm{K}_{\mathrm{ATP}}$ channel subunits) as well as the impact of 
p.Trp325Arg on their expression (Fig. $7 \mathrm{~g}-\mathrm{j}$ ). SLC30A8 transcript levels showed strong positive correlation with expression of all candidate genes ( $G C G, \mathrm{p} \leq 1.3 \times 10^{-7}, P C S K 1, p$ $\leq 1.5 \times 10^{-7}, P C S K 2, \mathrm{p} \leq 4.6 \times 10^{-10}, C P E, \mathrm{p} \leq 3.2 \times 10^{-6}, K C N J 11, \mathrm{p} \leq 7.1 \times 10^{-7}$, and $A B C C 8, p \leq 1.6 \times 10^{-11}$ ) except INS. The protective p.Trp325 allele showed a trend (nonsignificant) of decreased $S L C 30 A 8$ expression $(\mathrm{p}=0.053)$ as well as genes involved in proinsulin processing such as $P C S K 1(\mathrm{p}=0.041), P C S K 2(\mathrm{p}=0.045)$ and $A B C C 8(\mathrm{p}=$ $0.049)$.

Taken together, these data suggest that the common T2D-protective allele (p.Trp325) may also improve the response to a glucose challenge (Fig. 2 and Fig. 3) by enhancing insulin secretion and possibly by reducing glucagon secretion in primary human islets.

\section{Discussion}

The current study demonstrates the strengths of using human models for studying the consequences of variants associated with human diseases. Although over $30 \mathrm{~T} 2 \mathrm{D}$ protective LoF or missense alleles in $S L C 30 A 8$ have been reported ${ }^{19}$, previous studies in rodents $6,17,20,21,22$, have failed to provide a mechanistic explanation for the protection. This human study robustly shows that enhanced insulin responsiveness to glucose, combined with enhanced proinsulin processing, contribute to the protection from T2D. As all LoF carriers had the common risk p.Arg325 allele on the same haplotype, the effect of the LoF allele was most evident when compared against p.Arg 325 carriers, emphasizing the importance of considering the genetic background of human LoF carriers.

In our human iPSC-derived beta-like cells, two different LoF alleles (p.Arg138* and p.Lys34Serfs50*) show a clear reduction in $S L C 30 A 8$ expression, suggesting that NMDinduced haploinsufficiency is likely a common mechanism for rare LoF alleles in SLC30A8. Although a recent study showed increased zinc transporter activity for the common risk variant p.Arg $325^{23}$, it should be kept in mind that the common p.Trp325Arg locus is complex, as another 3' UTR variant (rs3802177) is in strong linkage disequilibrium with p.Trp325Arg. This variant (rs3802177) may potentially affect SLC30A8 transcription/ translation, further modulating the effect of p.Trp325Arg on ZnT8 function.

Interestingly, in our human iPSC-based model of SLC30A8 LoF mutations (p.Arg138* and p.Lys34Serfs50*), loss of ZnT8 protein decreased the formation of $I N S^{+}$cells. In support of this, we observed decreased expression of multiple genes associated with beta cell development (including $P D X 1$ ) and changes in expression of genes involved in WNT signaling (including TCF7L2) after partial ZnT8 loss (knockdown) in the human EndoC$\beta \mathrm{H} 1$ cell line. However, insulin content was not affected, suggesting that partial ZnT8 loss is unlikely to affect beta-cell development. In support of this, complete ZnT8 null mice were viable with preserved beta-cell function $6,9,10$. We have not been able to examine this in humans, as we did find any human homozygous for SLC30A8 LoF alleles.

Whilst data from all our sub-studies are consistent with increased insulin secretion, the precise molecular mechanisms for the involvement of zinc and zinc transporters remain elusive. 
Data derived from the human $\beta$-cell line show a clear decrease in intracellular zinc content and a trend towards a reduction in co-secreted granular zinc after ZnT8 loss. These observations are consistent with previous rodent studies demonstrating that loss of ZnT8 function reduces total islet zinc content (including $\mathrm{R} 138^{*}$ mice ${ }^{10}$ ) as well as free $\mathrm{Zn}^{2+}$ in the cytosol and granules $6,9,17,24$. The LoF p.Arg138* and p.Lys34Serfs50* alleles in humans are likely to exert similar effects on intracellular and granular zinc concentrations. In the present study, over-expression of the truncated p.Arg138* protein in INS-1 cells did not result in changes in cytosolic zinc concentrations, which is consistent with haploinsufficiency. In contrast, a recent human study showed that the T2D risk p.Arg325 allele was associated with higher islet zinc concentrations ${ }^{25}$. In support of a potential role for zinc in the development of diabetes, $\mathrm{Zn}^{2+}$ plays an important role as regulator of cellular excitability ${ }^{26}$ in the central nervous system (CNS). In beta-cells, $\mathrm{Zn}^{2+}$ induced inhibition of L-type voltage-gated $\mathrm{Ca}^{2+}$ channels could result in inhibition of insulin secretion ${ }^{27}$, whilst, $\mathrm{Zn}^{2+}$ has been reported to directly activate $\mathrm{K}_{\mathrm{ATP}}$ channel currents ${ }^{28}$. The down regulation of the $\mathrm{K}_{\mathrm{ATP}}$ channel subunits genes following SLC3OA8 knockdown suggests that there is coordinated expression of these genes. It is therefore tempting to hypothesize that $S L C 30 A 8$ expression may contribute to the normal $\mathrm{K}_{\mathrm{ATP}}$ channel density in both $\beta$ and a cells, but this remains to be demonstrated. The combined consequence of a reduction in $\mathrm{K}_{\mathrm{ATP}}$ channel gene expression and a reduction in para/autocrine electrophysiological regulation (on calcium and $\mathrm{K}_{\mathrm{ATP}}$ channels activities) by $\mathrm{Zn}^{2+}$ could contribute to enhanced insulin secretion as well as reducing glucagon secretion at low glucose levels. Indeed, a similar effect on alpha cells secretory capacity has been observed upon inhibition of $\mathrm{K}_{\mathrm{ATP}}$ channel activity using tolbutamide ${ }^{29}$.

$\mathrm{Zn}^{2+}$ is essential for proper insulin crystallization, and loss of $\mathrm{ZnT} 8$ results in reduced dense core granules and increased granule diameter possibly due to more non-crystalline insulin which is expected to be released rapidly during exocytosis $6,17,30$. The increased basal insulin secretion seen in the human $\beta$-cell line following SLC30A8 knockdown and also reported in islets from Slc30a8 null mice ${ }^{17}$, who also display impaired insulin crystallization, suggests that there is preferential release of non-crystalline insulin. It has also been suggested that increased insulin clearance could explain the decrease in circulating insulin concentrations ${ }^{20}$. However, we did not find any support for changes in insulin clearance in human p.Arg $138^{*}$ carriers or p.Arg 138* mice on HFD, nor did we see any effect on the incretin hormone GLP-1 in humans.

In contrast to the consistent effect of human p.Arg 138* and p.Trp325Arg on proinsulin processing, studies in mice following the loss of ZnT8 have reported inconsistent effects on proinsulin processing, ranging from inhibitory effects ${ }^{20,21}$ to beneficial effects in transgenic Trp325Arg mice on HFD ${ }^{22}$. The reasons for this remain unclear, but since blocking of the insulin receptor prevents the positive effect on insulin processing in p.Arg $138 *$ mice $^{10}$, it is possible that insulin receptor signaling modulates the effect of loss of ZnT8 on proinsulin processing.

The most reproducible finding in all sub-studies of $S L C 30 A 8$ loss was enhanced glucosestimulated insulin secretion associated with increased conversion of proinsulin to C-peptide and insulin. Carriers of the p.Trp325 T2D protective allele also showed a similar phenotype, 
consistent with previous published studies reporting impaired proinsulin conversion in carriers of the risk p.Arg325 allele ${ }^{31,32}$. It has been suggested that it takes some time for insulin to mature and become biologically active ${ }^{33}, 34$. It is therefore possible that the pronounced effect of the LoF T2D- protective allele at 20 and 40 minutes of the test meal could reflect an effect on the time-course of insulin maturation.

Our study has both strengths and limitations. Among its strengths are the comprehensive studies in humans recalled by their genotype in a region of the world where the p.Arg $138^{*}$ allele is enriched. The complementary studies in both human cell lines as well as the humanized mice can also be considered as a strength. A limitation is the inconclusive measurements of zinc in different cellular compartments and characterization of insulin granule cargo. This part will require the development of better intracellular sensors of zinc which will enable these studies in the future.

In conclusion, our data consistently demonstrate that heterozygosity for a LoF allele p.Arg138* and homozygosity for a common allele p.Trp325Trp of SLC3OA8 are associated with increased insulin secretion capacity and a lower risk of developing T2D and in the absence of any on-target adverse events. Therefore, ZnT8 remains an appealing safe target for antidiabetic therapies preserving $\beta$-cell function.

\section{Online Methods}

\section{Human study population}

The Botnia Study has been recruiting patients with T2D and their family members in the area of five primary health care centers in western Finland since 1990. Individuals without diabetes at baseline (relatives or spouses of patients with T2D) have been invited for followup examinations every 3-5 years ${ }^{11}$. The Prevalence, Prediction and Prevention of diabetes (PPP)-Botnia Study is a population-based study in the same region including a random sample of 5,208 individuals aged 18 to 75 years from the population registry ${ }^{38}$. Diabetes Registry Vaasa (DIREVA) is regional diabetes registry of $>5000$ diabetic patients from Western Finland (Botnia region) ${ }^{39}$. In the current study, we included $>14,000$ individuals (Botnia family study=5,678, PPP=4,862, and DIREVA=3,835). All participants gave their written informed consent and the study protocol was approved by the Ethics Committee of Helsinki University Hospital, Finland (the Botnia studies) and the Ethics Committee of Turku University Hospital (DIREVA). Study participants and their clinical measurements in the Verona Newly Diagnosed Diabetes Study (VNDS, Extended Data Fig. 3c) are described in the Supplementary Note.

\section{Oral Glucose Tolerance Test (OGTT, Fig. 3), test meal (Fig. 2 and Extended Data Fig. 2), and Intravenous Glucose Tolerance Test (IVGTT, Extended Data}

Fig. 3)-Subjects maintained a weight-maintaining diet and avoided vigorous exercise for 3 days prior to the OGTT or test meal, which were performed after an overnight fast. Height, weight, hip and waist circumferences, fat percentage (\%, bioimpedance analyzer) and blood pressure (sitting, 3 measurements after 5 minutes rest) were measured. The participants ingested $75 \mathrm{~g}$ dextrose (in a couple of minutes, OGTT) or a $526 \mathrm{kcal}$ mixed meal (in 10 minutes, test-meal: $76 \mathrm{~g}$ carbohydrate, $17 \mathrm{~g}$ protein and $15 \mathrm{~g}$ fat). Blood samples were drawn 
from an antecubital vein for plasma (P-) glucose and serum (S-) insulin and C-peptide at 0, 30, 120 minutes during the OGTT; for P-glucose, P-glucagon, S-insulin, S-C-peptide, Szinc, and total S-GLP-1 at 0, 20, 40, 70, 100, 130, 160 and 190 minutes during the test meal. Test meal samples for S-FFA were collected at 0, 40 and 120 minutes and for S-proinsulin at 0, 20, 40 and 130 minutes, respectively. Urine was collected between $0-70$ and $70-190$ minutes for the determination of glucose and zinc excretion during the test meal. Details about IVGTT is provided in the Supplementary Note.

Biochemical measurements (Fig. 2, 3 and Extended Data Fig. 3a-b)-See the Supplementary Note for details, corrected insulin response (CIR) was calculated for test meal (at 20 minutes) and OGTT (at 30 minutes) using the formula $\operatorname{CIR}(\mathrm{t})=\operatorname{Ins}(\mathrm{t}) \cdot 100 /$ [Gluc(t) • (Gluc(t)-3.89)], where Ins(t) and Gluc(t) are insulin (in mU/L) and glucose concentrations (in $\mathrm{mmol} / \mathrm{L}$ ) at sample time point (minutes) ${ }^{40}$. Estimation of Insulin clearance index was done on the model-based estimation of glucose-, insulin- and C-peptide curves during the test meal using the equation $\mathrm{AUC}_{\mathrm{ISR}} /\left[\mathrm{AUC}_{\mathrm{ins}}+\left(\mathrm{I}_{\mathrm{basal}}-\mathrm{I}_{\text {final }}\right) \cdot \mathrm{MRT}_{\mathrm{ins}}\right]$, where $\mathrm{AUC}_{\mathrm{ISR}}$ is the area under the curve of insulin secretion rate, $\mathrm{AUC}_{\mathrm{ins}}$ is the area under the curve of insulin concentration, $\mathrm{I}_{\text {final }}$ is insulin concentration at the end, and $\mathrm{I}_{\text {basal }}$ insulin concentration at the beginning of the study ${ }^{41} . \mathrm{MRT}_{\mathrm{ins}}$ is the mean residence time of insulin, and was assumed to be 27 minutes as reported previously ${ }^{42}$.

Genetic Association Analysis-The genotype data were obtained as described in the Supplementary Note. All the quantitative traits were inversely normally transformed before the analyses. The family-based recall study included only non-diabetic subjects during test meal. Association analysis of rare p.Arg $138 *$ with glycemic indices obtained during familybased recall test meal study (left and middle panel of Fig. 2, Extended Data Fig. 2 and Supplementary Table 6) was performed using family-based association analyses (orthogonal model together with 100,000 Monte-Carlo permutations) adjusting for age, sex, BMI, and additionally other covariates (genotype of p.Trp325Arg for middle panel only) as implemented in QTDT (v2.6.1, https://csg.sph.umich.edu/abecasis/QTDT/) ${ }^{35}$. Association analysis of common p.Trp325Arg with glycemic indices obtained during family-based recall test meal study (right panel of Fig. 2, Extended Data Fig. 2, and Supplementary Table 6) was performed using QFAM family-based association test with 100,000 permutations to correct for any family structure as implemented in PLINK ${ }^{36}$ (--qfam-total, --mperm 100,000, http:// zzz.bwh.harvard.edu/plink/contact.shtml\#cite). The association analysis of p.Arg138* and p.Trp325Arg with glycemic traits during OGTT studies including only non-diabetic individuals (Fig. 2, Supplementary Table 4 and Supplementary Table 5) and IVGTT (Extended Data Fig. 3) was performed using mixed linear model considering genetic relatedness among samples as implemented in GCTA (v1.91, http://cnsgenomics.com/ software/gcta) ${ }^{37}$. The fixed-effects meta-analysis of all OGTT studies (Supplementary Table 5) were performed using METAL software package (http://www.sph.umich.edu/csg/ abecasis/metal/) ${ }^{43}$. The linear mixed model (adjusting for genetic relatedness) also used for $\mathrm{T} 2 \mathrm{D}$ association analysis. 
iPSC generation, differentiation and genome editing (Fig. 4 and Extended Data Fig. 4 and 5)

iPSC generation and maintenance-The human induced pluripotent stem cell line (hiPSC) SB Ad3.1 was previously generated and obtained through the IMI/EU sponsored StemBANCC consortium via the Human Biomaterials Resource Centre, University of Birmingham (http://www.birmingham.ac.uk/facilities/hbrc). Human skin fibroblasts were obtained from a commercial source (Lonza CC-2511, tissue acquisition number 23447). They had been collected from a Caucasian donor with no reported diabetes with fully informed consent and with ethical approval from the National Research Ethics Service South Central Hampshire research ethics committee (REC 13/SC/0179). The fibroblasts were reprogrammed to pluripotency as previously described ${ }^{44}$ and were subjected to the following quality control checks: SNP-array testing via Human CytoSNP-12v2.1 beadchip (Illumina \#WG-320-2101), DAPI-stained metaphase counting and mFISH, flow cytometry for pluripotency markers (BD Biosciences \#560589 and 560126), and mycoplasma testing (Lonza \#LT07-118).

\section{CRISPR-Cas9 mediated generation of p.Arg138* and p.Lys34Serfs50* human induced pluripotent stem cell line-Several guide RNAs (gRNAs) were designed} using MIT CRISPR tool (http://crispr.mit.edu/) to target near exon 2 and exon 3 of SLC30A8 (ENST00000456015). The gRNAs were also subjected to an additional BlastN search (www.ensembl.org) to confirm specificity and identified no additional off-target sites. To generate SLC30A8-p.Arg138*, the target site for CRISPR-Cas9 mutagenesis (Supplementary Table 8) was sub-cloned into the $B s b I$ restriction sites within the gRNA structure in the $\mathrm{pX} 330^{45}$ plasmid that was previously modified to contain a puromycin selection cassette. A single strand oligonucleotide repair template for homology-directed repair (HDR) was synthesized by Eurogentec, stabilized by addition of a phosphorothioate linkage at the 5' end, and contained two nucleotide changes: i) the T2D-protective nonsense mutation at codon-138 (c.412C > T, p.Arg 138*), which also mutated the PAM sequence, and ii) a silent mutation at codon-139 (c.417A $>$ T, p.Ala139Ala) to introduce an $A l u I$ restriction site for genotyping. Human iPSCs were co-transfected with the SLC30A8-px330-puromycin resistant vectors and the HDR repair template using Fugene6 according to manufacturer's guidelines (Promega \#E2691). Following transient puromycin-selection, single clones were picked and expanded as described previously ${ }^{46}$. Genotyping PCR was performed using primers (primer pair 1, Supplementary Table 8) to amplify targeted region followed by $A l u I$ restriction digest. Successfully targeted clones were confirmed via Sanger sequence and monoallelic sequencing was performed by TA-cloning (pGEM®-T Easy Vector System; Promega \#A1360) of the PCR amplicons. From 96 clones, 11 clones were heterozygous for p.Arg138*, four of which contained indels in the non-targeted allele. The CRISPR-Sham hiPSC control line (p.Arg138Arg) was generated from hiPSC cells that went through the CRISPR pipeline without being edited at the SLC30A8 locus. The two p.Arg138* clones (A3 and B1) and the unedited control line (p.Arg138Arg) passed quality control checks that included repeat chromosome counting and pluripotency testing. Both p.Arg $138 *$ clones were heterozygous for the c.412C>T, p.Arg138*, while the silent variant at codon-139 (c.417A>T, p.Ala139Ala) was present in both alleles. Description of p.Lys34Serfs50* human induced pluripotent stem cell line generation is provided in the Supplementary Note. 
In vitro differentiation of hiPSCs towards Beta-like cells-Directed differentiation of hiPSCs towards beta-like cells was performed using a previously published protocol ${ }^{12,47}$. hiPSCs were seeded on Growth Factor Reduced Matrigel-coated CellBind 12-well tissue culture plates (Corning \#356230 \& \#3336) at a cell density of $1.3 \times 10^{6}$ in mTesR1 (Stem Cell Technologies \#05850) with $10 \mu \mathrm{M}$ Y-27632 dihydrochloride (Abcam \#ab120129). The following morning, hiPSCs were fed mTesR1 media $>4$ hours before starting the seven-stage differentiation protocol described in the Supplementary Note.

\title{
Quantification of SLC30A8 gene expression in Beta-like Cells derived from
} CRISPR-edited hiPSCs-Expression of $S L C 30 A 8$ was measured at the end of stage 7 using quantitative PCR (qPCR). Briefly, RNA was extracted using TRIzol Reagent (Life Technologies \#15596026) according to manufacturer's instructions. cDNA was amplified using the GoScript Reverse Transcription Kit (Promega \#A5000). qPCR was performed using $40 \mathrm{ng}$ of cDNA, TaqMan ${ }^{\circledR}$ Gene Expression Master Mix (Applied Biosystems \#4369017) and primer/probes for SLC30A8 (Hs00545182_m1) or the housekeeping gene TBP(Hs00427620_m1). Gene expression was determined using the $\Delta \Delta C T$ method by first normalizing to $T B P$ and then to the control p.Arg138Arg (n=7-13 wells from three differentiations).

\begin{abstract}
Allele-specific SLC30A8 expression in beta-like Cells derived from CRISPRedited hiPSCs-Stage 7 cells were treated with $100 \mu \mathrm{g} / \mathrm{mL}$ cycloheximide (Sigma \#C4859) or DMSO (Sigma \#D2650) for four hours at $37^{\circ} \mathrm{C}^{48}$ before or RNA and cDNA synthesis as above. Allele specific expression was measured using the QX10 Droplet Digital PCR System and C1000 Touch Thermal Cycler according to manufacturer's guidelines (Bio-Rad). Custom primers (primer pair 2, Supplementary Table 8) and probes for the detection of p.Arg 138* variant were designed using Primer3Plus (Applied Biosystems): FAM probe (R138; CT, Supplementary Table 8)), VIC probe (X138; TT, Supplementary Table 8). The specificity of the probes was confirmed by ddPCR using R138 or X138 templates (Extended Data Fig. 5). Results were analyzed using Quanta Soft software (Bio$\mathrm{Rad})$ and presented as a ratio of wildtype to HDR-edited allele expression ( $\mathrm{n}>4$ wells from two differentiations).
\end{abstract}

Western blot of ZnT8-We used highly specific antibody for ZnT8 as developed by Merriman et al. ${ }^{49}$ (Supplementary Note). The signal detected correspond of a protein of 30-34 KDa consistent with previous publication ${ }^{49}$.

\section{Gene expression measurements in Beta-like Cells derived from CRISPR-} edited hiPSCs-Allele-specific SLC3OA8 expression by targeted RNA sequencing (Fig. 4e and Extended Data Fig. 5c) and other transcript expression in hiPSC-derived BLCs by RNAscope ${ }^{\circledR}$ (Extended Data Fig. 4e-g) have been detailed in the Supplementary Note.

\section{EndoC- $\beta \mathrm{H} 1$ culture (Fig. 5)}

The results obtained in EndoC- $\beta \mathrm{H} 1$ are from two distinct teams (Helsinki and Oxford) with different batches of EndoC- $\beta \mathrm{H} 1$ cultures. Here, we report both methods and specify for each experiment the origin of the culture (Helsinki or Oxford). EndoC- $\beta \mathrm{H} 1$ cells were cultured in 
medium and grown on a matrix as described previously ${ }^{50}$ and tested negative for mycoplasma.

SLC30A8 knockdown in EndoC- $\beta$ H1 cells-In Oxford, EndoC- $\beta$ H1 cells were transfected with $10 \mathrm{nM}$ siRNA (either SMARTpool ON-TARGETplus SLC30A8 or scramble [Dharmacon \#L-007529-01]) and Lipofectamine RNAiMAX (Life Technologies \#13778-075) according to manufacturer's instructions for a total of 72 hours. In Helsinki, EndoC- $\beta$ H1cells were transfected using Lipofectamin RNAiMAX (life technologies). 20nM siRNA ON-TARGETplus siRNA SMARTpool for human SLC30A8 gene (Dharmacon; L-007529-01) and ON-TARGET plus Non-targeting pool (siNT or Scramble) (Dharmacon; D-001810-10-05) were used following the protocol as described previously ${ }^{51}$. Cells were harvested $96 \mathrm{~h}$ post-transfection for further studies.

Insulin secretion measurements in EndoC- $\beta \mathrm{H} 1$ cells-In Oxford, cells were subjected to static insulin secretion assays $72 \mathrm{~h}$ after siRNA transfection as described previously ${ }^{51}$, apart from the following modifications: cells were stimulated for $1 \mathrm{~h}$ with 1 $\mathrm{mM}$ glucose, $20 \mathrm{mM}$ glucose, $1 \mathrm{mM}$ glucose $+200 \mu \mathrm{M}$ tolbutamide, or $20 \mathrm{mM}$ glucose $+500 \mu \mathrm{M}$ diazoxide. Insulin levels were measured in both supernatants and cells using the Insulin (human) AlphaLISA Detection Kit and EnSpire Alpha Plate Reader (Perkin Elmer \#AL204C and \#2390-0000, respectively). Cell count per well was measured via CyQUANT Direct Cell Proliferation Assay (Thermo Fisher\# C35011). Data are presented as insulin secretion normalized to percentage of insulin content from Control condition. RNA extraction, cDNA synthesis, and qRT-PCR were performed as above ( $S L C 30 A 8$ gene expression in CRISPR-edited hiPSCs derived beta like cell section) to determine $S L C 30 \mathrm{~A} 8$ knockdown and expression of the $\mathrm{K}_{\mathrm{ATP}}$ channel genes (ABCC8Hs01093752_m1 and KCNJ11 Hs00265026_s1; ThermoFisher Scientific). In Helsinki, EndoC- $\beta$ H1 cells were transfected with $20 \mathrm{nM}$ siRNA and Scramble control ${ }^{52}$. Following $96 \mathrm{~h}$ of siRNA transfection, cells were incubated overnight in $1 \mathrm{mM}$ glucose containing EndoC- $\beta \mathrm{H} 1$ culture medium. One hour prior to glucose stimulation assay, the media was replaced by $\beta$ KREBS (Univercell Biosolution S.A.S., France) without glucose. Cells were stimulated with 16.7 $\mathrm{mM}$ glucose and $50 \mathrm{mM} \mathrm{KCl}$ (Sigma-Aldrich) in $\beta$ KREBS for 30 minutes at $37^{\circ} \mathrm{C}$ in a $\mathrm{CO}_{2}$ incubator. The cells were then washed and lysed with TETG (Tris pH8, Trito X-100,

Glycerol, $\mathrm{NaCl}$ and EGTA) solution (Univercell Biosolution S.A.S., France) for the measurement of total insulin content. Secreted and intracellular insulin were measured using a commercial human insulin Elisa kit (Mercodia AB, Uppsala, Sweden) as per manufacturer's instructions (Helsinki).

\section{Electrophysiological measurements in EndoC- $\beta \mathrm{H} 1$ cells (Oxford)-SLC3OA8} was knocked down in EndoC- $\beta \mathrm{H} 1$ as above. $\mathrm{K}^{+}$ATP channel conductance was measured in a perforated patch whole cell configuration, and patch-clamped using an EPC 10 amplifier and HEKA pulse software. KREBS extracellular solution was perfused in at $32^{\circ} \mathrm{C}$ and contained: $138 \mathrm{mM} \mathrm{NaCl}, 3.6 \mathrm{mM} \mathrm{KCl}, 0.5 \mathrm{mM} \mathrm{MgSO}_{4}, 10 \mathrm{mM}$ HEPES, $0.5 \mathrm{mM} \mathrm{NaH}_{2} \mathrm{PO}_{4}, 5 \mathrm{mM}$ $\mathrm{NaHCO}_{3}, 1.5 \mathrm{mM} \mathrm{CaCl}_{2}, 1 \mathrm{mM}$ glucose and $100 \mu \mathrm{M}$ Diazoxide (Sigma-Aldrich \#D9035). The perforation of the membrane was achieved using an intra-pipette solution containing: $0.24 \mathrm{mg} / \mathrm{mL}$ amphotericin B, $128 \mathrm{mM}$ K-gluconate (Sigma \#Y0000005 and G4500 
respectively), $10 \mathrm{mM} \mathrm{KCl}, 10 \mathrm{mM} \mathrm{NaCl}, 1 \mathrm{mM} \mathrm{MgCl} 2,10 \mathrm{mM}$ HEPES, pH 7.35 (KOH). Conductance data are normalized to cell size and presented as $\mathrm{pS}_{\mathrm{p}} \mathrm{pF}^{-1}$. Expression of $A B C C 8, K C N J 11, B 2 M$, and $T B P$ were measured via qPCR as above (SLC3OA8 gene expression in CRISPR-edited hiPSCs derived beta like cell section).

Other assays in EndoC- $\beta \mathrm{H} 1$ cells-Other assays including insulin and proinsulin secretion and content, Immunoblotting (ZnT8, CPE, PC2, Phospho-AKT-Ser473, and AKT), Cell viability assay (MTT), Zinpyr-1 based Zinc staining, monitoring of stimulated zinc secretion using ZIMIR in EndoC- $\beta \mathrm{H} 1$ cells and RNA (mRNAs) sequencing of EndoC- $\beta \mathrm{H} 1$ cells are described in the Supplementary Note.

Data Analyses-Data are reported as mean (SEM). Statistical analyses were performed using Prism 6.0 (GraphPad Software). All parameters were analyzed using Mann-Whitney test or one sample $t$ test as indicated (Two-tailed, Fig. 5). See the Supplementary Note for description of RNA sequencing analysis.

\section{Mouse Model (Fig. 6)}

Animals-All procedures were conducted in compliance with protocols approved by the Regeneron Pharmaceuticals Institutional Animal Care and Use Committee. The

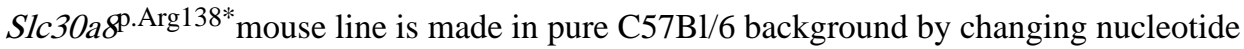
409 from $\mathrm{T}$ into $\mathrm{C}$ in exon 3 , which changes the arginine into a stop codon ${ }^{10}$. The mutated allele has a self-deleting neomycin selection cassette flanked by loxP sites inserted at intron 3 , deleting $29 \mathrm{bp}$ of endogenous intron 3 sequence. Mice were housed (up to five mice per cage) in a controlled environment (12-h light/dark cycle, 22C, 60-70\% humidity) and fed ad libitum with either chow (Purina Laboratory 23 Rodent Diet 5001, LabDiet) or high-fat diet (Research Diets, D12492; 60\% fat by calories) starting at age of 20 weeks. All data shown are compared to their respective WT littermates.

Glucose Tolerance Test-Mice were fasted overnight ( $16 \mathrm{hr}$ ) followed by oral gavage of glucose (Sigma) at $2 \mathrm{~g} / \mathrm{kg}$ body weight. Blood samples were obtained from the tail vein at the indicated times and glucose levels were measured using the AlphaTrak2 glucometer (Abbott). Submandibular bleeds for insulin were done at 0, 15, and 30 minutes post-injection in a separate experiment to not interfere with glucose levels.

Hormone measurements-Submandibular bleeds of either overnight fasted (16 h) animals were done in the morning. Plasma insulin or proinsulin was analyzed with the mouse insulin/proinsulin EIA (Mercodia AB, Uppsala, Sweden), and C-peptide with the mouse C-peptide EIA (ALPCO). All EIAs were performed according to the manufacturer's instructions.

Data Analyses for mouse studies-Data are reported as mean (SEM). Statistical analyses were performed using Prism 8.0 (GraphPad Software). All parameters were analyzed by two-way ANOVA (repeated measurements) combined with Sidak's multiple comparison test, or Mann Whitney test (two-tailed) as indicated (Fig. 6). 


\section{Expression of p.Arg138* mutation in INS1E (Extended Data Fig. 8)}

Details are provided in the Supplementary Note.

\section{Human Pancreatic Islets (Fig. 7)}

Experiments on primary human pancreatic islets were independently performed in two places 1) Oxford and 2) Lund university diabetes center (LUDC)

Human pancreatic islets from Oxford-Human pancreatic islets were isolated from deceased donors under ethical approval obtained from the human research ethics committees in Oxford (REC: 09/H0605/2, NRES committee South Central-Oxford B). All donors gave informed research consent as part of the national organ donation program. Islets were obtained from the Diabetes Research \& Wellness Foundation Human Islet Isolation Facility, OCDEM, University of Oxford. All methods and protocols using human pancreatic islets were performed in accordance with the relevant guidelines and regulations in the UK (Human Tissue Authority, HTA). For in vitro insulin secretion, islets were pre-incubated in Krebs-Ringer buffer (KRB) containing $2 \mathrm{mg} / \mathrm{mL}$ BSA and $1 \mathrm{mM}$ glucose for $1-\mathrm{h}$ at $37^{\circ} \mathrm{C}$, followed by 1-hour stimulation in KRB supplemented with $6 \mathrm{mM}$ glucose. Insulin content of the supernatant was determined by radioimmunoassay (Millipore UK Ltd, Livingstone, UK) as described previously ${ }^{53}$.

Human pancreatic islets from LUDC-Human pancreatic islets were obtained from the Human Tissue Laboratory (Lund University, www.exodiab.se/home) in collaboration with The Nordic Network for Clinical Islet Transplantation Program (www.nordicislets.org) ${ }^{54,55}$. All the islet donors provided their consent for donation of organs for medical research and the procedures were approved by the ethics committee at Lund University (Malmö, Sweden, permit number 2011263). Islet preparation for cadaver donors, their purity check and counting procedure have been described previously ${ }^{56}$. Static in vitro insulin secretion assay from 91 islets (non-diabetic individuals) was performed as described previously ${ }^{56,57}$. Briefly, six batches of 12 islets per donor were incubated for $1 \mathrm{~h}$ at $37^{\circ} \mathrm{C}$ in Krebs Ringer bicarbonate (KRB) buffer in presence of $1 \mathrm{mM}$ or $16.7 \mathrm{mM}$ glucose. Independently, KCL based insulin secretion assay was performed by incubating them in $70 \mathrm{mM} \mathrm{KCl}$ together with $1 \mathrm{mM}$ or $16.7 \mathrm{mM}$ glucose in a subset of islets in different batches. Insulin concentrations in the extracts were measured using a radioimmunoassay kit (Euro-Diagnostica, Malmö, Sweden). The Association of p.Trp325Arg genotype with expression of $S L C 30 A 8$ and other candidate genes was performed using RNA sequencing from islets of 139 non-diabetic individuals as described previously ${ }^{54,55}$ (see the Supplementary Note).

\section{Statistics}

Detail information regarding statistical tests (Two tailed; Mann-Whitney test or one sample test, genetic association; mixed model or linear regression or quantitative transmission disequilibrium tests) used for each sub-study has been provided in their respective method section or with figure legends. Sample size details could be found in figure legends and Source Data. For electrophysiology, $\mathrm{n}$ are representative of the number of siRNA transfected cells measured from several passages of the same EndoC- $\beta \mathrm{H} 1$ cell batch. For hormone 
secretion data, $\mathrm{n}$ are representative of independent biological replicates from independent passages of cells.

\section{Extended Data}



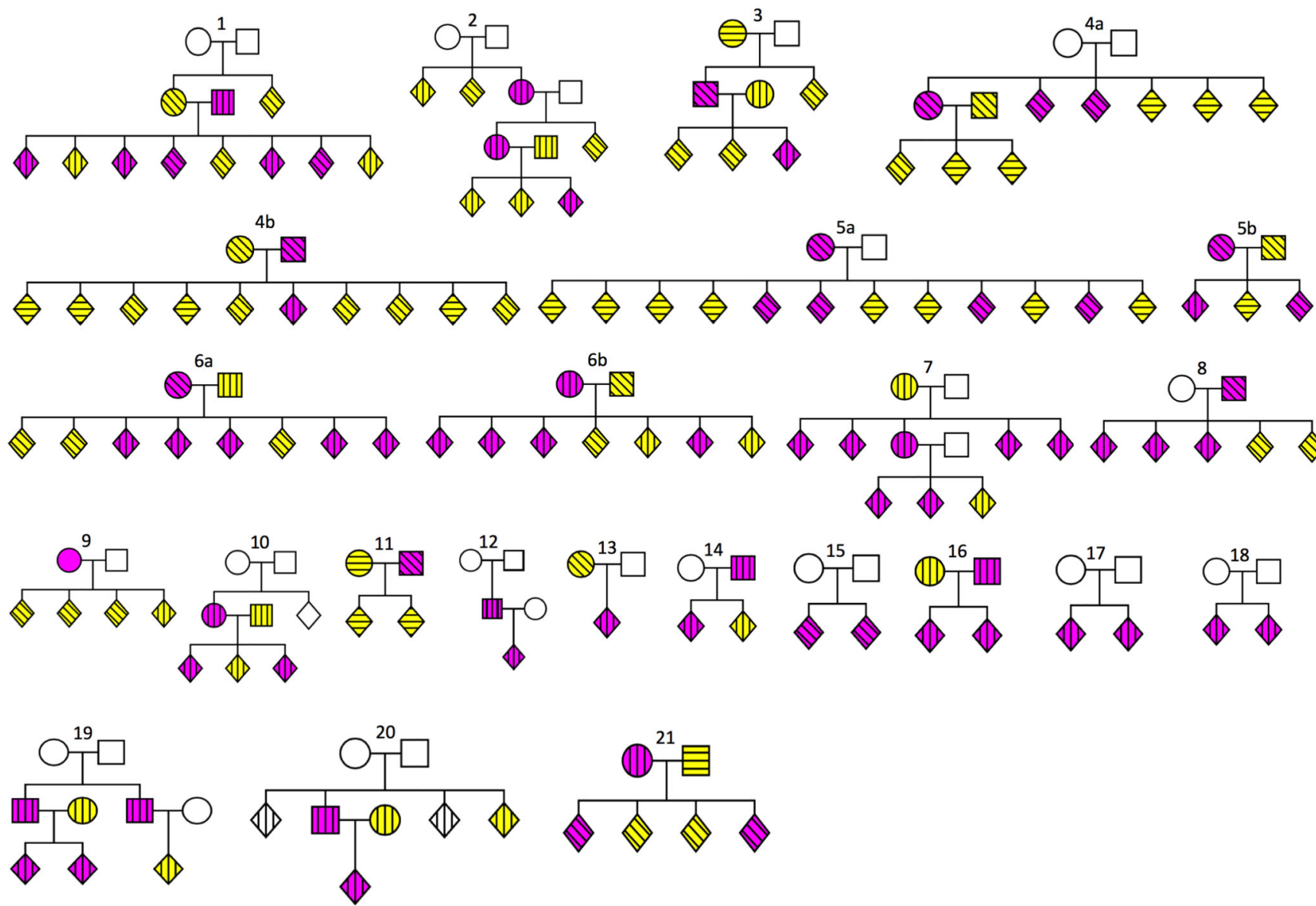

Extended Data Fig. 1. Families of SLC30A8-p.Arg138* carriers involved in genotype-based recall for human in vivo study.

Families ( $\geq 2$ members per family) of SLC30A8-p.Arg138* carriers participated in genotype-based recall (test meal) study. To protect anonymity of the carriers, the gender of the offspring is not revealed and some pedigrees have been split to smaller nuclear families. The carrier status of p.Arg138* is shown by yellow (p.Arg138Arg) or magenta (p.Arg138*), that of p.Trp325Arg by vertical (p.Arg325Arg), horizontal (p.Trp325Trp) or diagonal (p.Trp325Arg) lines. The white color (with no lines) represents individuals with unknown status for genotype and phenotype. 

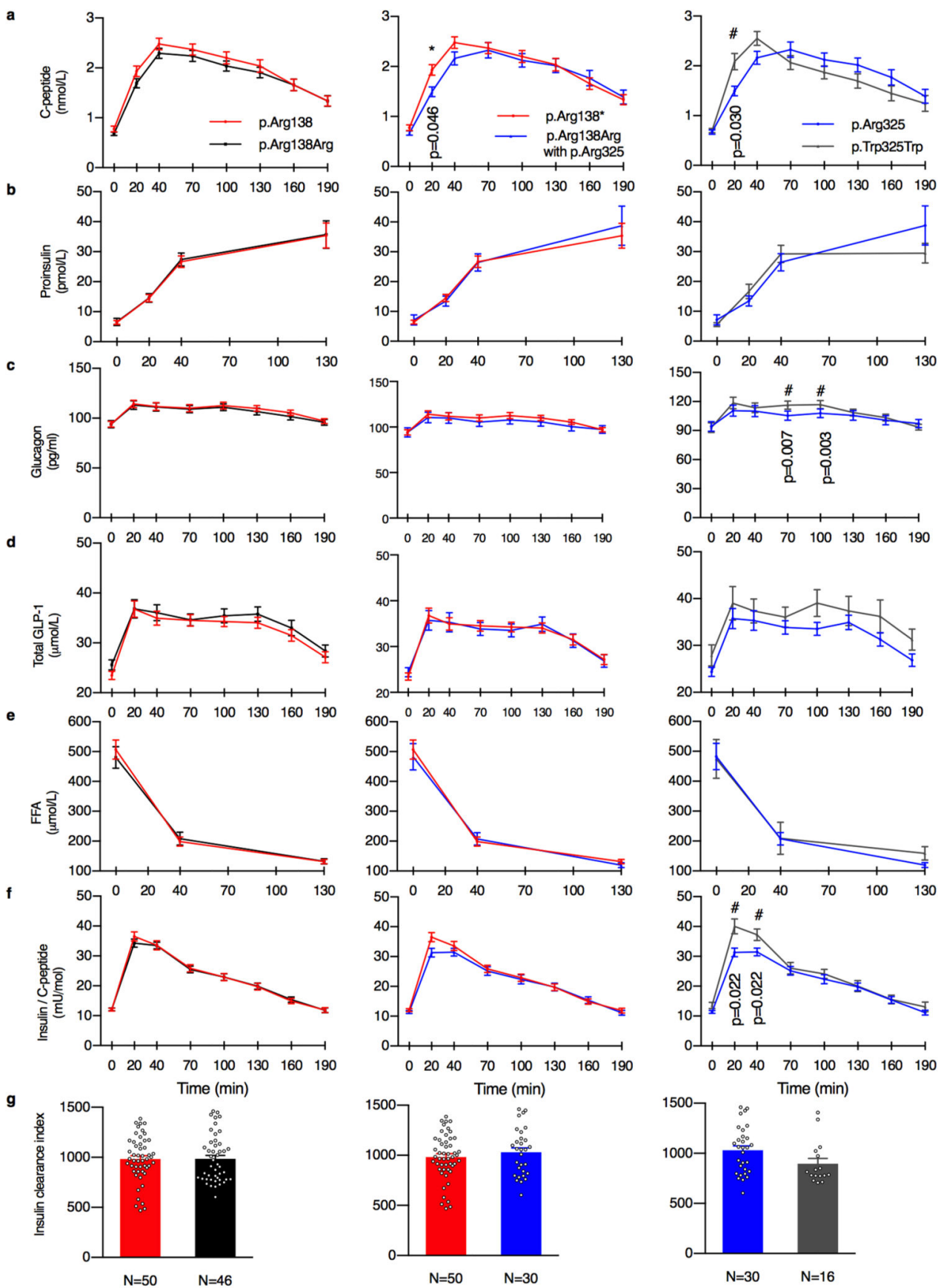

Extended Data Fig. 2. Association of $S L C 30 A 8$ p.Arg138* and p.Trp325Arg with free fatty acids, hormones, and insulin clearance during test meal.

Association of $S L C 30 A 8$ p.Arg 138* and p.Trp35Arg variant with a, serum (S)-C-peptide b, S-proinsulin c, plasma (P)-Glucagon d, Total S-GLP-1 e, S-free fatty acid (FFA) concentrations $\mathbf{f}$, Insulin-C-peptide ratio and $\mathbf{g}$, model-based insulin clearance index during test meal. Left panel: Carriers (red, n=50-54) vs. non-carriers (black, n=37-47) of p.Arg138*. Middle panel: Carriers of p.Arg138* (red, n=50-54) vs. p.Arg138Arg having the common risk variant p.Arg325 (blue, $\mathrm{n}=25-31$ ). Right panel: Carriers of p.Trp325Trp (grey, 
$\mathrm{n}=12-16$ ) vs. $\mathrm{p} \cdot \operatorname{Arg} 325$ (blue, $\mathrm{n}=25-31$ ). Exact numbers used for genetic association analysis are available in Source Data files. Data are Mean \pm SEM; A star $(* \mathrm{p}<0.05, * * \mathrm{p}<0.01)$ indicates significance in family based association (using QTDT35) after 100,000 permutations, adjusted for age, sex and BMI for left panel and age, sex, BMI and genotype of p.Trp325Arg for middle panel. A hash sign (\# p < 0.05) indicates significance in QFAM (as implemented in PLINK ${ }^{36}$ ) test using 100,000 permutations (see Methods). 

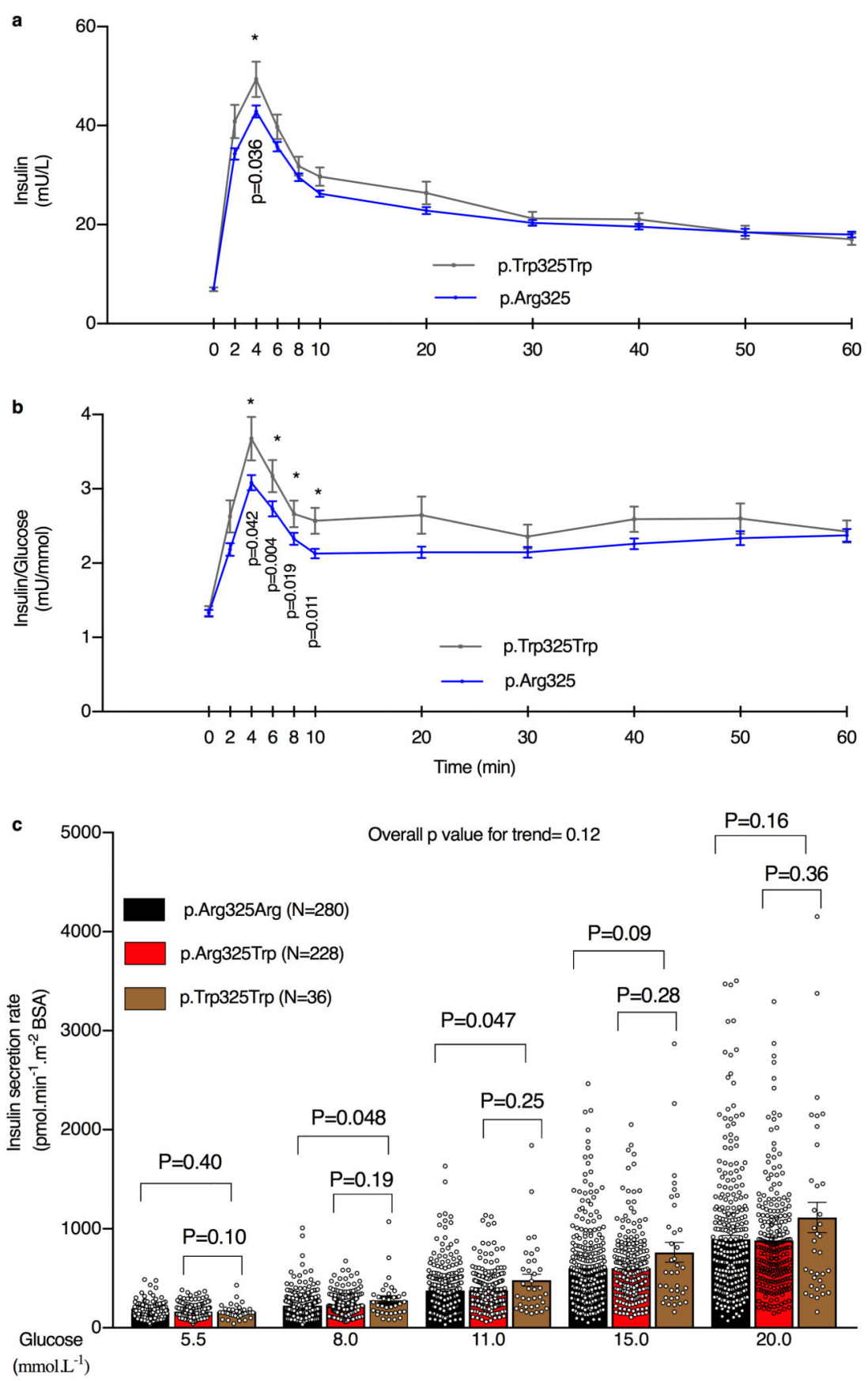

Extended Data Fig. 3. Effect of p.Trp325Arg genotype on insulin secretion during intravenous glucose tolerance test (IVGTT) and $\beta$-cell sensitivity to glucose during OGTT

a-b, (a) Serum (S-) insulin concentrations, p.Trp325Trp (grey, n=116) and p.Arg325 (blue, $\mathrm{n}=733$ ), and (b) S-insulin -plasma glucose ratio, p.Trp325Trp (grey, n=86) and p.Arg325 (blue, $\mathrm{n}=458$ ) during IVGTT. Data are Mean \pm SEM. Analysis was performed using mixed model adjusting for age, sex, BMI and genetic relatedness. * $\mathrm{p}<0.05$

c, $\beta$-cell sensitivity to glucose is presented as insulin secretion rate in response to plasma glucose during oral glucose tolerance test (OGTT) in people with newly diagnosed type 2 
diabetes ${ }^{58}$. Data are Mean \pm SEM. Analysis was performed using a generalized linear model (log-transformed data) for repeated measures, adjusting for age, sex and BMI. 
SLCBOA8
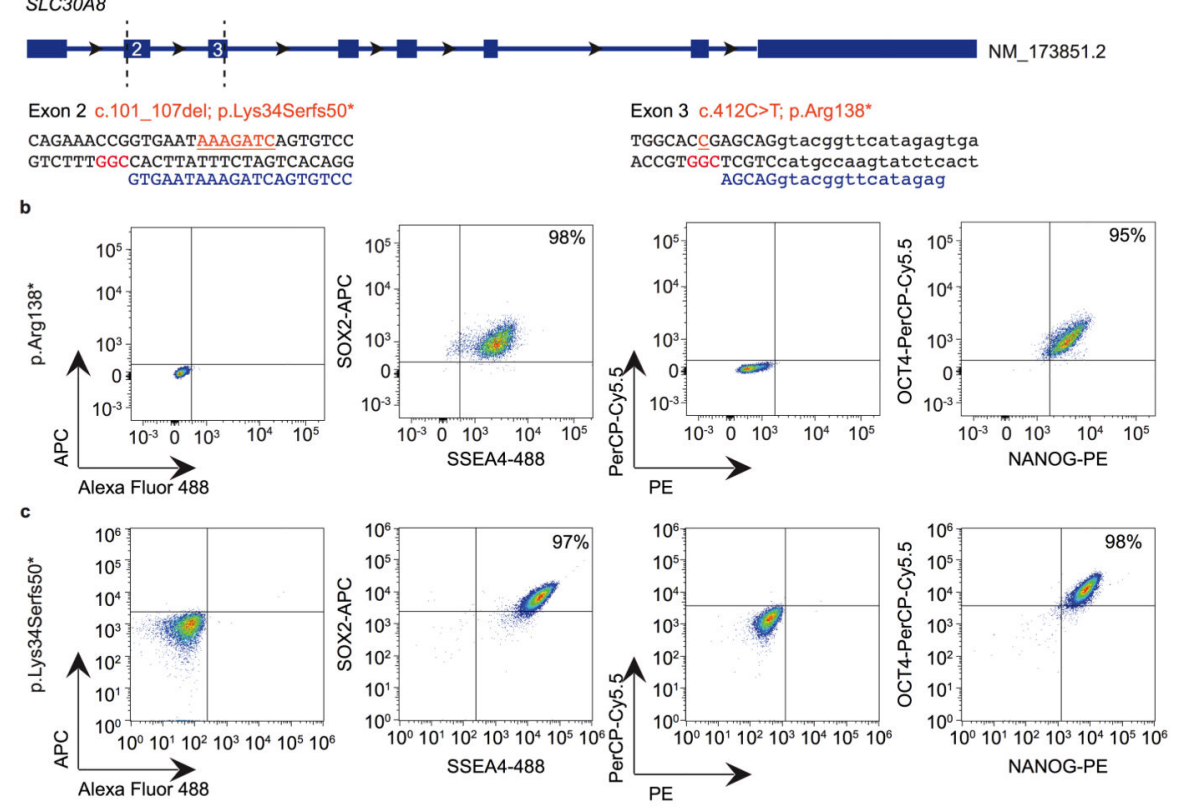

d
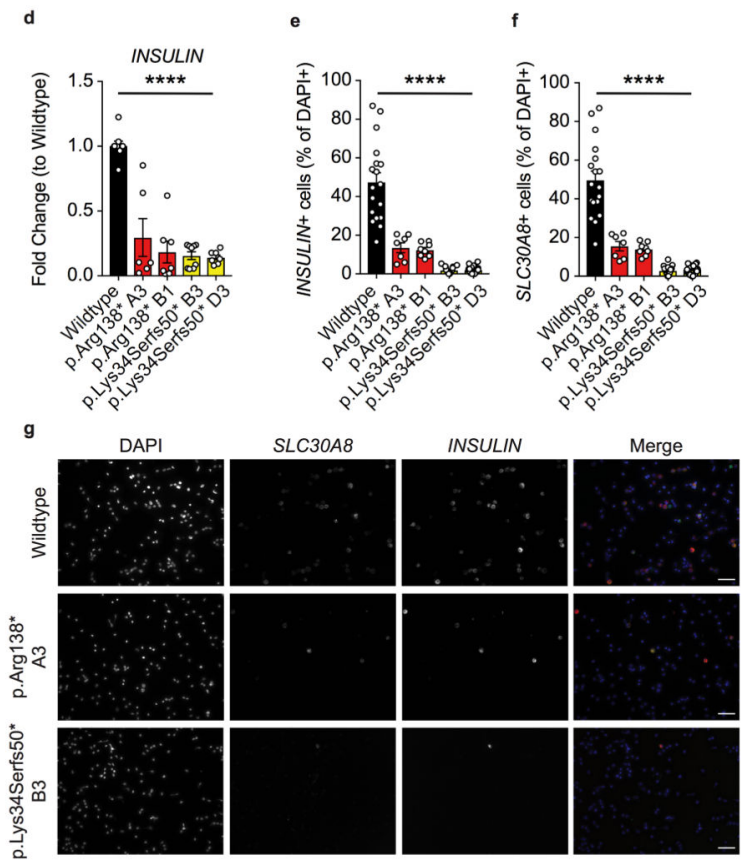

Extended Data Fig. 4. Generation of $S L C 30 A 8$-p.Lys34Serfs50* and $S L C 30 A 8$-p.Arg138* hiPSC lines.

a, CRISPR-Cas9 strategy to generate SLC30A8-p.Lys34Serfs50* (Exon 2) and SLC30A8p.Arg138* (Exon 3) hiPSC lines. Orange font highlights the nucleotide changes: c.

101-107del; p.Lys34Serfs50* and c.412C>T; p.Arg138*. The gRNA (blue font) and PAM sequences (red font) are indicated on the partial genomic sequence of $S L C 30 A 8$. b-c, FACS data from undifferentiated b, SLC30A8-p.Arg138* and c, SLC30A8-p.Lys34Serfs50* iPSCs and relevant isotype controls using antibodies against: OCT3/4, SSEA, SOX2, and NANOG. 
d, Expression of INSULIN in hiPSC-derived beta-like cells. Black bars represent p.Arg138Arg control cells, red bars represent p.Arg 138*, and yellow bars represent p.Lys34Serfs50*. ( $\mathrm{n}=6-8$ wells from three differentiations) e-g, RNAscope analysis of the number of e, INSULIN- and f, SLC3OA8-transcript positive cells in hiPSC-derived beta-like cells. 7-21 image fields were quantified and presented as \% of DAPI+ cells. Representative images used for quantification shown in $\mathbf{g}$ (scale bar $=50 \mu \mathrm{m}$ ). Data are presented as Mean \pm SEM. Statistical analysis was performed using the one-way ANOVA and Tukey's multiple comparison test $(\mathrm{n}=5-9$ wells from three differentiations, $* * * * \mathrm{p}<0.0001)$. 

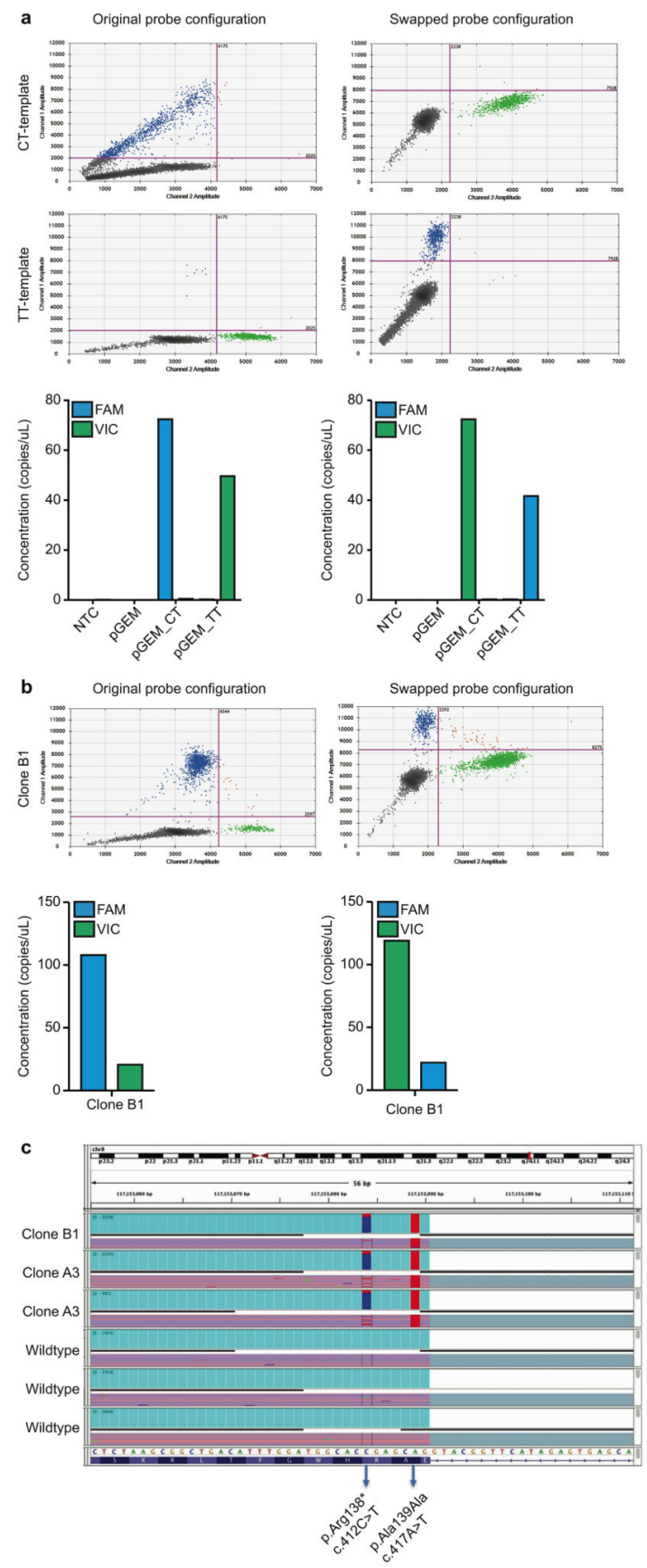

Extended Data Fig. 5. Confirmation of the ddPCR probe specificity and target $S L C 30 A 8$ mRNA sequencing.

a, R138 (pGEM_CT) and X138 (pGEM_TT) sequences were inserted in pGEM vector and used as template for digital droplet PCR. Original probe configuration confirmed specificity as R138 droplets were only detected by FAM (CT-FAM; channel 1) and X138 droplets were only detected by VIC (TT-VIC; channel 2). In the swapped probe configuration, FAM and VIC probes were swapped and ddPCR was performed using pGEM_CT or pGEM_TT as template. b, Detection of R138 allele (Channel 1) and X138 allele (Channel 2) using cDNA from the heterozygous hiPSC-derived beta-like cells (B1 clone) as a template. In the 
swapped probe configuration, FAM and VIC probes were swapped and ddPCR was performed using cDNA from hiPSC-derived beta-like cells as template. $\mathbf{c}$, Depicting the unique sequencing reads coverage at p.Arg $138^{*}$ and p.Ala139Ala obtained by SLC3OA8 target mRNAs sequencing in edited clones (B1 and A3) and unedited cells (wildtype). 
a<smiles>[C]#C</smiles><smiles></smiles>

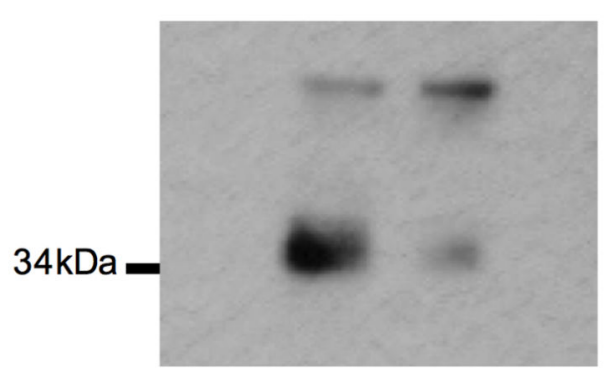

C

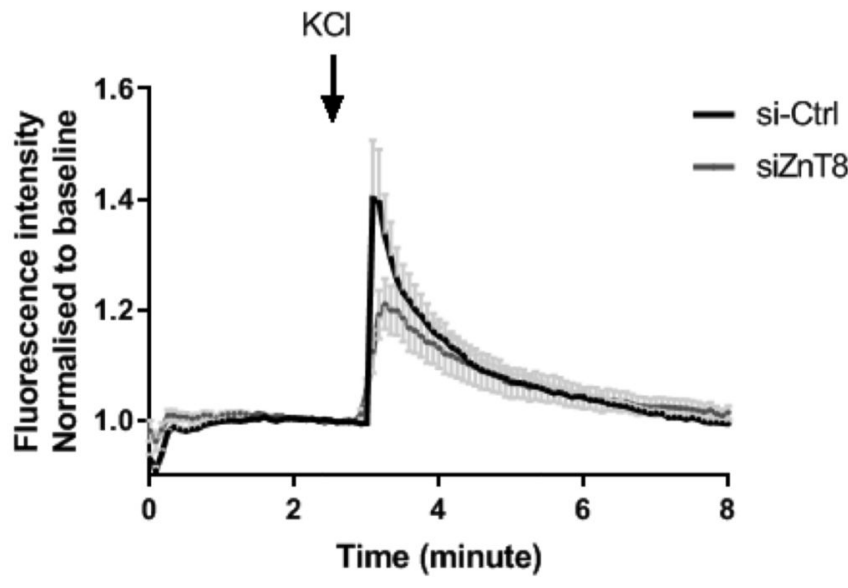

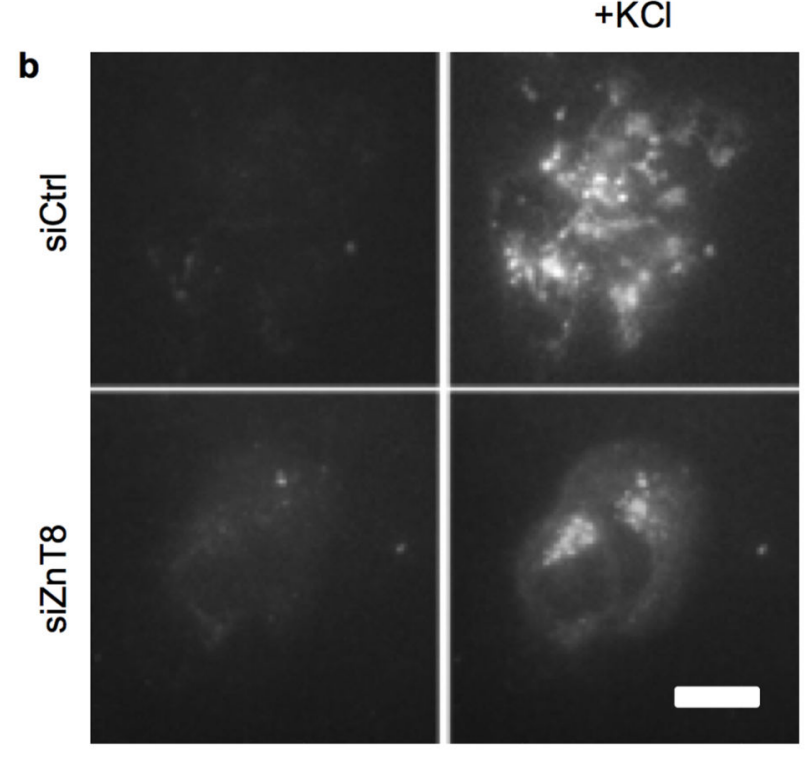

d

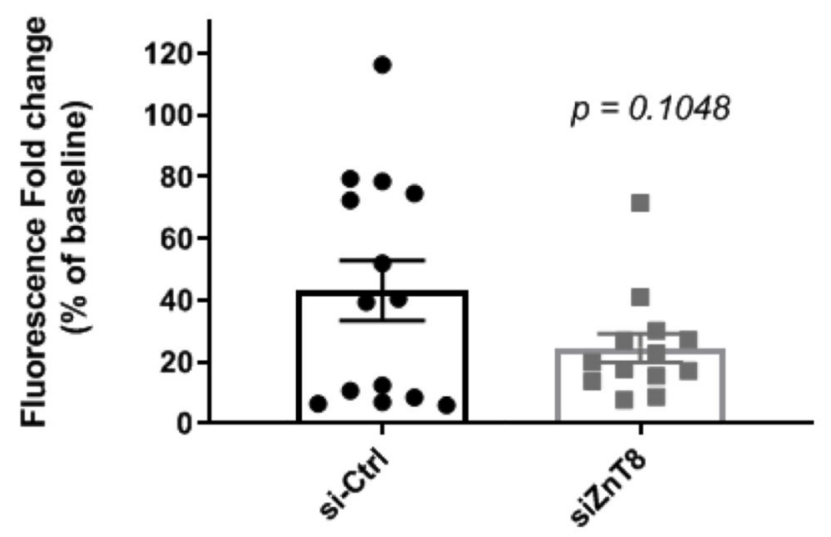

Extended Data Fig. 6. Silencing of $\mathrm{ZnT8}$ tends to lower granule $\mathrm{Zn}^{2+}$ content.

EndoC- $\beta \mathrm{H} 1$ cells were transfected with siRNA control (siCtrl) or targeted against SLC30A8

(siZnT8) for 72 hours prior to imaging. a, ZnT8 knock-down was confirmed at the protein level by Western-blot. b, Control cells were incubated for $20 \mathrm{~min}$. with the membranetargeted zinc probe ZIMIR ${ }^{59}$ to monitor zinc secretion after cell stimulation with $20 \mathrm{mM}$ $\mathrm{KCl}$ using total internal reflection of fluorescence (TIRF) microscopy (see accompanying movies, Supplementary video 1-4). c, Fluorescence intensity at the membrane was monitored upon time and traces obtained were averaged for cells transfected with siCtrl (14 cells) or with siZnT8 (14 cells). d, Fluorescence intensity increase due to zinc secretion after stimulation with $\mathrm{KCl}$ was determined for each cell. An outlier data point in the siZnT8 condition, likely to reflect release from a non-silenced cell, was excluded by Grubb's test and statistical significance determined by Student's t-test (Graph Pad Prism 7.0). Scale bar 
in $\mathbf{b}, 5 \mu \mathrm{m}$. Blots have been cropped and corresponding full blots are available in Source Data files. 

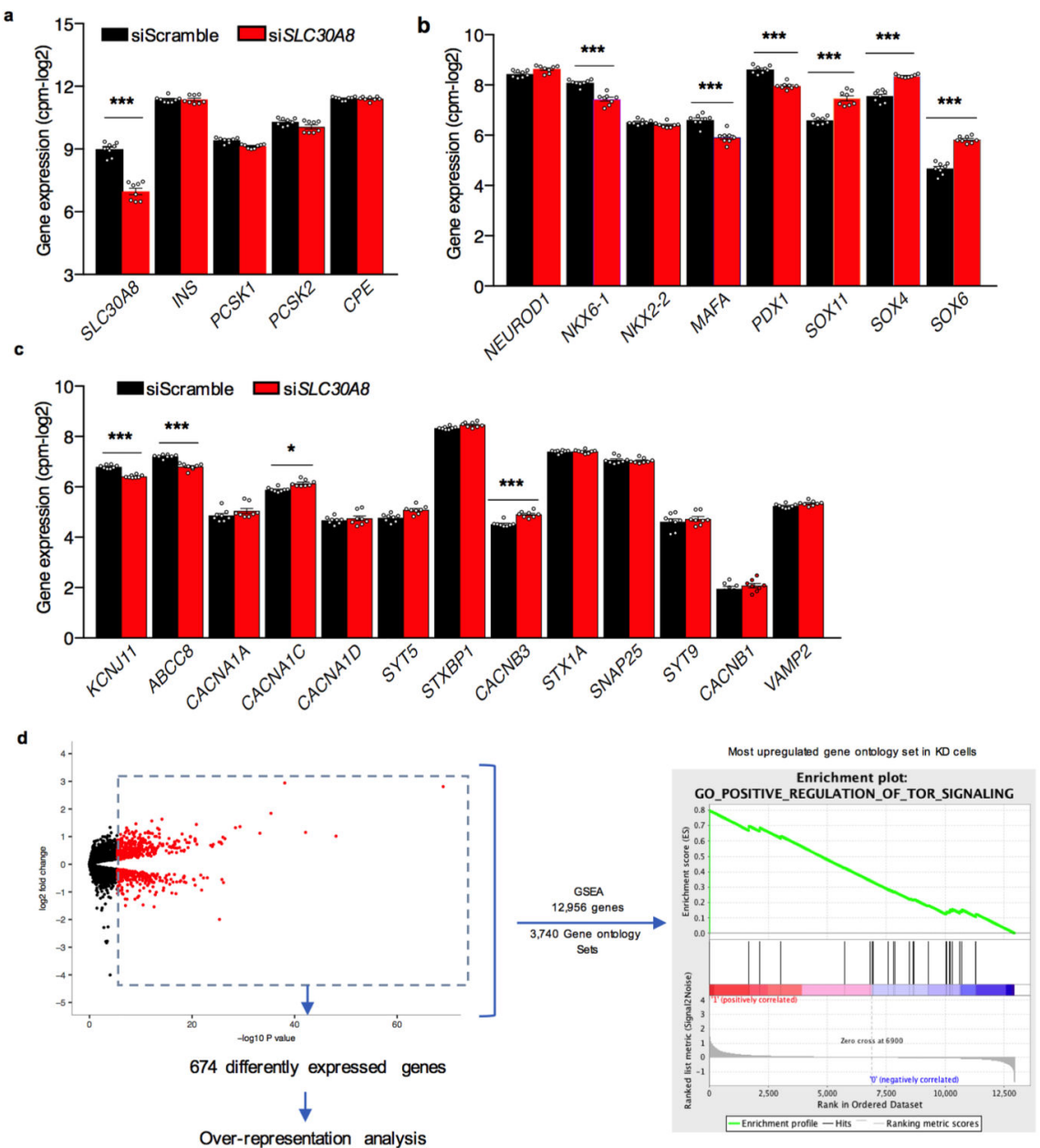

1

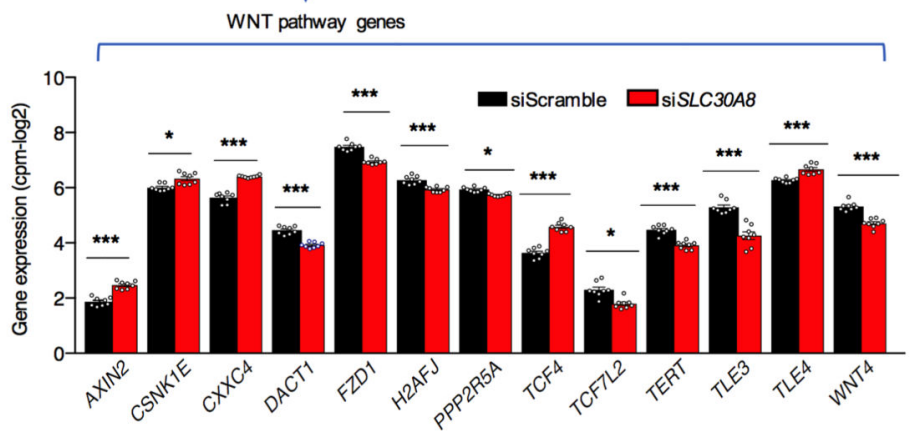

Extended Data Fig. 7. RNA (mRNAs) sequencing of $S L C 30 A 8$ knock down and control EndoC$\beta H 1$ cells.

Effect of SLC30A8 knock down (KD) on expression of genes involved in a, proinsulin processing, $\mathbf{b}$, insulin production and $\beta$-cell development, $\mathbf{c}, \beta$-cell excitability and insulin exocytosis (Supplementary Data Set 1). d, Over-representation analysis of differentially expressed genes (red dots) and depiction of most enriched WNT pathway genes (Supplementary Data Set 1) along with a gene set enrichment analysis (GSEA) considering all expressed genes. ${ }^{*} \mathrm{p}<0.05,{ }^{* * *} \mathrm{p}<0.001$; Differential expression analysis was done using 
sequencing reads count based data using method similar to Fisher's Exact test as implemented in edgR software package ${ }^{60}$ and further corrected for multiple testing using Bonferroni correction (see Supplementary Note). 
a

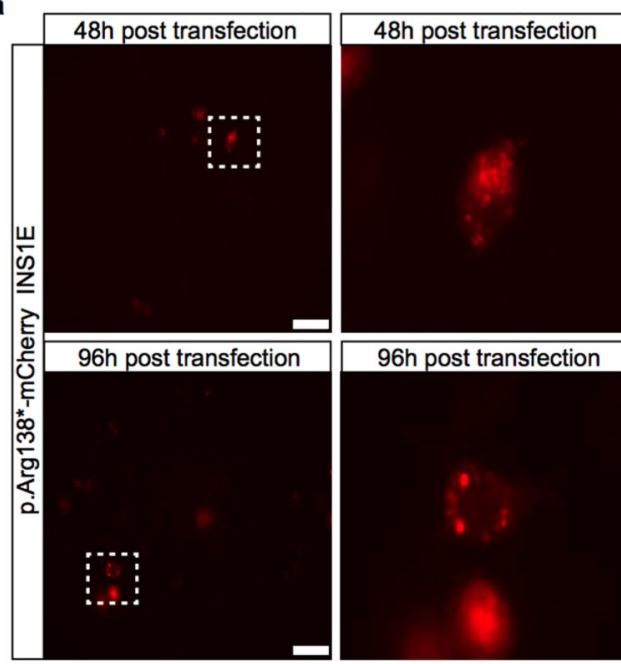

b
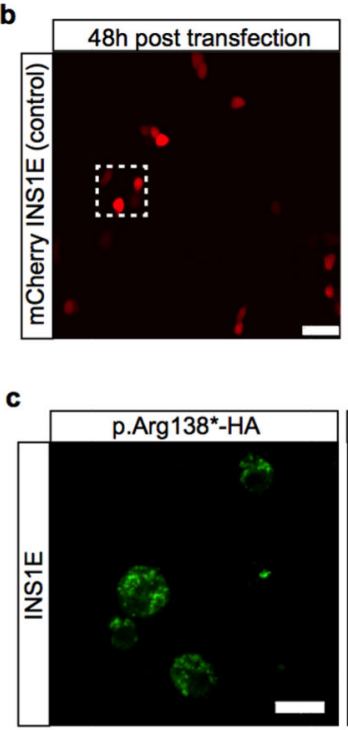

$48 \mathrm{~h}$ post transfection
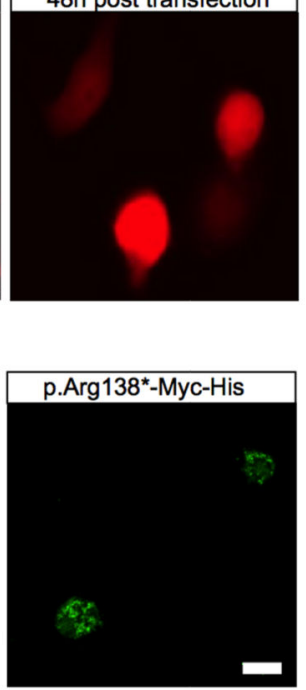

d

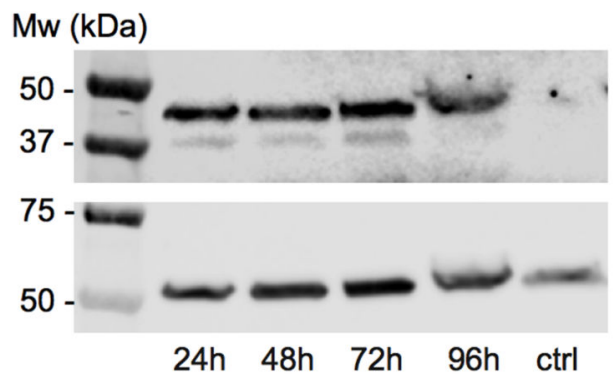

e

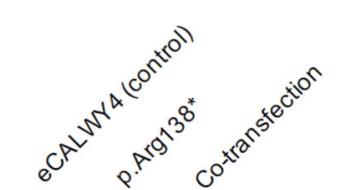

$\mathbf{f}$
o
$\frac{m}{5}$
$\frac{5}{5}$
0

C-Myc

DAPI

Merged

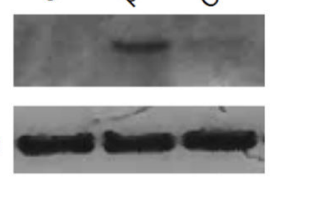

Tubulin
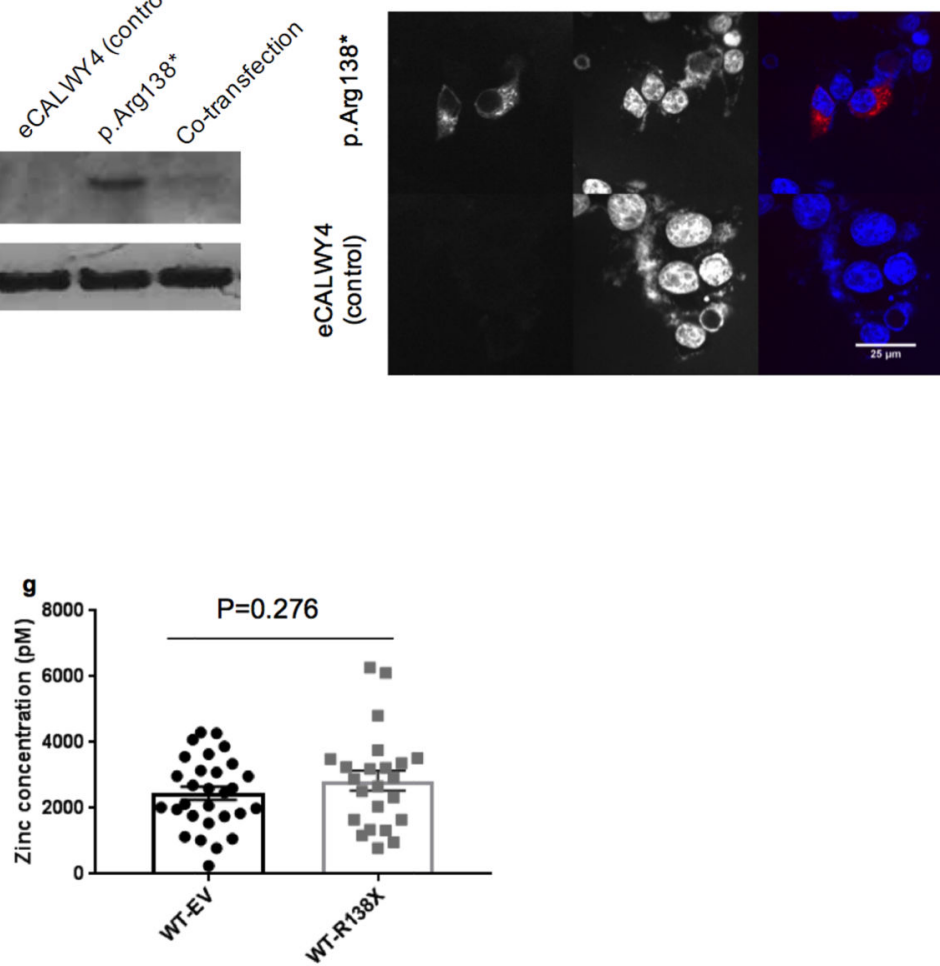

Extended Data Fig. 8. Expression and localization of p.Arg138* and impact on cytosolic free $\mathrm{Zn}^{2+}$ concentrations in cultured INS1 $\beta$-cells.

a-d, Rat INS1e cells were transiently transfected with p.Arg138*-mCherry fusion construct followed by fluorescence microscopy imaging and immunodetection. a, Fusion protein localized to distinct subcellular compartments in INS1e cells at $48 \mathrm{~h}$ and $96 \mathrm{~h}$ after transfection. b. Expression of mCherry in control INS1e cells indicated cytoplasmic localization. c, Control experiments with immunostaining of p.Arg138* with HA or MycHis (both are significantly smaller additions than mCherry) confirmed localization of fusion proteins to distinct subcellular compartments in the INS1e cells. d, Immunological detection (anti-mCherry) of the fusion protein at indicated time points after transfection confirms protein expression and indicate protein stability. Tubulin is used as control. e-f, INS1(832/13) cells were transfected constructs expressing p.Arg138*-Myc-His or eCALWY-4, or co-transfected with both, followed by (e) immunostaining or (f) 
immunofluorescence imaging at $24 \mathrm{~h}$ post-transfection using anti-c-Myc antibody. $\mathbf{g}$, Cytosolic free $\mathrm{Zn}^{2+}$ concentrations in INS-1 (832/13) cells. Data are combined from three fully independent experiments. Scale bars are $50 \mu \mathrm{m}(\mathbf{a}, \mathbf{b}), 10 \mu \mathrm{m}(\mathbf{c})$ and $25 \mu \mathrm{m}$ (f). Blots have been cropped and corresponding full blots are available in Source Data files.

\section{Supplementary Material}

Refer to Web version on PubMed Central for supplementary material.

\section{Acknowledgements}

We thank the Botnia Study Group for recruiting and studying the participants, Jens Juul Holst for measuring GLP-1 concentrations, and Linda Boselli, $\mathrm{PhD}$, for carrying out mathematical modelling of the OGTT studies. We thank Dr. Dax Fu (Department of Physiology, The Johns Hopkins School of Medicine, Baltimore, Maryland) for providing monoclonal anti-ZnT8 antibody. We thank Dr Wen-hong Li (Departments of Cell Biology and of Biochemistry, University of Texas Southwestern Medical Center, Dallas) for providing zinc probe ZIMIR. We thank Erqian Na for her help with the mouse immunohistochemistry and histology, and Catherine Green and the Chromosome Dynamics \& Genome Engineering Cores at the Wellcome Centre for Human Genetics for support with karotyping and genome editing (funded by the Welcome Trust grant 203141). We thank the Sequencing Unit core facility at FIMM Technology Centre supported by University of Helsinki and Biocenter Finland. The Botnia and The PPP-Botnia studies (L.G., T.T.) have been financially supported by grants from Folkhälsan Research Foundation, the Sigrid Juselius Foundation, The Academy of Finland (grants no. 263401, 267882, 312063 to L.G., 312072 to T.T., 317599 to O.P.D.), Nordic Center of Excellence in Disease Genetics, EU (EXGENESIS, EUFP7MOSAIC FP7-600914), Ollqvist Foundation, Swedish Cultural Foundation in Finland, Finnish Diabetes Research Foundation, Foundation for Life and Health in Finland, Signe and Ane Gyllenberg Foundation, Finnish Medical Society, Paavo Nurmi Foundation, Helsinki University Central Hospital Research Foundation, Perklén Foundation, Närpes Health Care Foundation and Ahokas Foundation, as well as by the Ministry of Education in Finland, Municipal Heath Care Center and Hospital in Jakobstad and Health Care Centers in Vasa, Närpes and Korsholm. The work described in this paper has been supported with funding from collaborative agreements with Pfizer Inc. as well as with Regeneron Genetics Center LLC. The work was also supported by Hjelt Foundation (L.G. and C.B.W.) and Rhapsody (L.G.). J.O.L. was supported by Vinnova - Sweden's Innovation Agency (2015-01549), Swedish Diabetes Foundation, Albert Påhlsson Foundation, Hjelt Foundations, Crafoord Foundation, Royal Physiographic Society in Lund, Swedish Foundation for Strategic Research (IRC15-0067), Swedish Research council (2009-1039, Strategic research area Exodiab); E.A. by Crafoord Foundation, Påhlsson Foundation, Swedish Research Council (Dnr: 2017-02688); O.H. by Lund University Diabetes Center, ALF, Crafoord foundation, Novo Nordisk foundation, Magnus Bergvall foundation, Påhlsson foundation, Diabetes Wellness and Swedish Diabetes Research Foundation; R.C.B. by Italian Ministry of University and Research (PRIN 2015373Z39_004) and University of Parma Research Funds; G.R. by a Wellcome Trust Senior Investigator Award (WT098424AIA), MRC Programme grants (MR/R022259/1, MR/J0003042/1, MR/L020149/1) and Experimental Challenge Grant (DIVA, MR/L02036X/1), MRC (MR/N00275X/1), Diabetes UK (BDA/11/0004210, BDA/15/0005275, BDA 16/0005485) and Imperial Confidence in Concept (ICiC) grants, and a Royal Society Wolfson Research Merit Award. ALG is a Wellcome Trust Senior Fellow in Basic Biomedical Science. M.I.M. and P.R. are Wellcome Senior Investigators. This work was funded in Oxford by the Wellcome Trust (095101 [ALG], 200837 [A.L.G.], 098381 [M.I.M.], 106130 [A.L.G., M.I.M.], 203141 (A.L.G., B.D., M.I.M.], 203141 [M.I.M.], 090531 [P.R.]), Medical Research Council (MR/L020149/1) [M.I.M., A.L.G., P.R.], European Union Horizon 2020 Programme (T2D Systems) [A.L.G.], and NIH (U01-DK105535; U01-DK085545) [M.I.M., A.L.G.]. The research was funded by the National Institute for Health Research (NIHR) Oxford Biomedical Research Centre (BRC) [A.L.G., M.I.M., P.R.]. The views expressed are those of the author(s) and not necessarily those of the NHS, the NIHR or the Department of Health.

\section{References}

1. Chabosseau P, Rutter GA. Zinc and diabetes. Arch Biochem Biophys. 2016; 611:79-85. [PubMed: 27262257]

2. Chimienti F, Devergnas S, Favier A, Seve M. Identification and cloning of a beta-cell-specific zinc transporter, ZnT-8, localized into insulin secretory granules. Diabetes. 2004; 53:2330-7. [PubMed: 15331542]

3. Flannick J, et al. Loss-of-function mutations in SLC30A8 protect against type 2 diabetes. Nat Genet. 2014; 46:357-63. [PubMed: 24584071] 
4. Parsons DS, Hogstrand C, Maret W. The C-terminal cytosolic domain of the human zinc transporter ZnT8 and its diabetes risk variant. FEBS J. 2018; 285:1237-1250. [PubMed: 29430817]

5. Sladek R, et al. A genome-wide association study identifies novel risk loci for type 2 diabetes. Nature. 2007; 445:881-5. [PubMed: 17293876]

6. Lemaire K, et al. Insulin crystallization depends on zinc transporter ZnT8 expression, but is not required for normal glucose homeostasis in mice. Proc Natl Acad Sci U S A. 2009; 106:14872-7. [PubMed: 19706465]

7. Pound LD, et al. Deletion of the mouse Slc30a8 gene encoding zinc transporter-8 results in impaired insulin secretion. Biochem J. 2009; 421:371-6. [PubMed: 19450229]

8. Wijesekara N, et al. Beta cell-specific Znt8 deletion in mice causes marked defects in insulin processing, crystallisation and secretion. Diabetologia. 2010; 53:1656-68. [PubMed: 20424817]

9. Mitchell RK, et al. Molecular Genetic Regulation of Slc30a8/ZnT8 Reveals a Positive Association with Glucose Tolerance. Mol Endocrinol. 2016; 30:77-91. [PubMed: 26584158]

10. Kleiner S, et al. Mice harboring the human SLC30A8 R138X loss-of-function mutation have increased insulin secretory capacity. Proc Natl Acad Sci. 2018; 115:E7642-E7649. [PubMed: 30038024]

11. Groop L, et al. Metabolic consequences of a family history of NIDDM (the Botnia study): evidence for sex-specific parental effects. Diabetes. 1996; 45:1585-93. [PubMed: 8866565]

12. Rezania A, et al. Reversal of diabetes with insulin-producing cells derived in vitro from human pluripotent stem cells. Nat Biotechnol. 2014; 32:1121-33. [PubMed: 25211370]

13. Miyaoka Y, Chan AH, Conklin BR. Using Digital Polymerase Chain Reaction to Detect SingleNucleotide Substitutions Induced by Genome Editing. Cold Spring Harb Protoc. 2016

14. Scharfmann R, et al. Development of a conditionally immortalized human pancreatic $\beta$ cell line. $\mathbf{J}$ Clin Invest. 2014; 124:2087-98. [PubMed: 24667639]

15. Li D, et al. Imaging dynamic insulin release using a fluorescent zinc indicator for monitoring induced exocytotic release (ZIMIR). Proc Natl Acad Sci USA. 2011; 108:21063-8. [PubMed: 22160693]

16. Hastoy B, et al. Electrophysiological properties of human $\beta$-cell lines EndoC- $\beta \mathrm{H} 1$ and $-\beta \mathrm{H} 2$ conform with human $\beta$-cells. 2017; doi: 10.1101/226282v1

17. Nicolson TJ, et al. Insulin storage and glucose homeostasis in mice null for the granule zinc transporter ZnT8 and studies of the type 2 diabetes-associated variants. Diabetes. 2009; 58:207083. [PubMed: 19542200]

18. Vinkenborg JL, et al. Genetically encoded FRET sensors to monitor intracellular Zn2+ homeostasis. Nat Methods. 2009; 6:737-40. [PubMed: 19718032]

19. Flannick J, et al. Exome sequencing of 20,791 cases of type 2 diabetes and 24,440 controls. Nature. 2019; 570:71-76. [PubMed: 31118516]

20. Tamaki M, et al. The diabetes-susceptible gene SLC30A8/ZnT8 regulates hepatic insulin clearance. J Clin Invest. 2013; 123:4513-24. [PubMed: 24051378]

21. Hardy AB, et al. Effects of high-fat diet feeding on Znt8-null mice: differences between $\beta$-cell and global knockout of Znt8. Am J Physiol Endocrinol Metab. 2012; 302:E1084-96. [PubMed: 22338079]

22. Li L, Bai S, Sheline CT. hZnT8 (Slc30a8) Transgenic Mice that Overexpress the R325W Polymorph Have Reduced Islet Zn2+ and Proinsulin Levels, Increased Glucose Tolerance After a High-Fat Diet, and Altered Levels of Pancreatic Zinc Binding Proteins. Diabetes. 2017; 66:551559. [PubMed: 27899481]

23. Merriman C, Huang Q, Rutter GA, Fu D. Lipid-tuned Zinc Transport Activity of Human ZnT8 Protein Correlates with Risk for Type-2 Diabetes. J Biol Chem. 2016; 291:26950-26957. [PubMed: 27875315]

24. Gerber PA, et al. Hypoxia lowers SLC30A8/ZnT8 expression and free cytosolic Zn2+ in pancreatic beta cells. Diabetologia. 2014; 57:1635-44. [PubMed: 24865615]

25. Wong WP, et al. Exploring the Association Between Demographics, SLC30A8 Genotype, and Human Islet Content of Zinc, Cadmium, Copper, Iron, Manganese and Nickel. Sci Rep. 2017; 7(1):473. [PubMed: 28352089] 
26. Vergnano AM, et al. Zinc dynamics and action at excitatory synapses. Neuron. 2014; 82:1101-14. [PubMed: 24908489]

27. Ferrer R, Soria B, Dawson CM, Atwater I, Rojas E. Effects of Zn2+ on glucose-induced electrical activity and insulin release from mouse pancreatic islets. Am J Physiol. 1984; 246:C520-7. [PubMed: 6372518]

28. Bloc A, Cens T, Cruz H, Dunant Y. Zinc-induced changes in ionic currents of clonal rat pancreatic -cells: activation of ATP-sensitive K+ channels. J Physiol. 2000; 529:723-34. [PubMed: 11118501]

29. Zhang Q, et al. Role of KATP channels in glucose-regulated glucagon secretion and impaired counterregulation in type 2 diabetes. Cell Metab. 2013; 18:871-82. [PubMed: 24315372]

30. Michael DJ, et al. Pancreatic beta-cells secrete insulin in fast- and slow-release forms. Diabetes. 2006; 55:600-7. [PubMed: 16505221]

31. Kirchhoff K, et al. Polymorphisms in the TCF7L2, CDKAL1 and SLC30A8 genes are associated with impaired proinsulin conversion. Diabetologia. 2008; 51:597-601. [PubMed: 18264689]

32. Majithia AR, et al. Association of the SLC30A8 missense polymorphism R325W with proinsulin levels at baseline and after lifestyle, metformin or troglitazone intervention in the Diabetes Prevention Program. Diabetologia. 2011; 54:2570-4. [PubMed: 21779873]

33. Jainandunsing S, et al. A stable isotope method for in vivo assessment of human insulin synthesis and secretion. Acta Diabetol. 2016; 53:935-944. [PubMed: 27552831]

34. Ivanova A, et al. Age-dependent labeling and imaging of insulin secretory granules. Diabetes. 2013; 62:3687-96. [PubMed: 23929935]

35. Abecasis GR, Cardon LR, Cookson WO. A general test of association for quantitative traits in nuclear families. Am J Hum Genet. 2000; 66:279-92. [PubMed: 10631157]

36. Purcell S, et al. PLINK: a tool set for whole-genome association and population-based linkage analyses. Am J Hum Genet. 2007; 81:559-75. [PubMed: 17701901]

37. Yang J, Lee SH, Goddard ME, Visscher PM. GCTA: a tool for genome-wide complex trait analysis. Am J Hum Genet. 2011; 88:76-82. [PubMed: 21167468]

38. Isomaa B, et al. A family history of diabetes is associated with reduced physical fitness in the Prevalence, Prediction and Prevention of Diabetes (PPP)-Botnia study. Diabetologia. 2010; 53:1709-13. [PubMed: 20454776]

39. Ahlqvist E, et al. Novel subgroups of adult-onset diabetes and their association with outcomes: a data-driven cluster analysis of six variables. Lancet Diabetes Endocrinol. 2018; 6:361-369. [PubMed: 29503172]

40. Sluiter WJ, Erkelens DW, Reitsma WD, Doorenbos H. Glucose tolerance and insulin release, a mathematical approach I. Assay of the beta-cell response after oral glucose loading. Diabetes. 1976; 25:241-4. [PubMed: 773721]

41. Mohandas C, et al. Ethnic differences in insulin secretory function between black African and white European men with early type 2 diabetes. Diabetes Obes Metab. 2018; 20:1678-1687. [PubMed: 29516668]

42. Navalesi R, Pilo A, Ferrannini E. Kinetic analysis of plasma insulin disappearance in nonketotic diabetic patients and in normal subjects. A tracer study with 125I-insulin. J Clin Invest. 1978; 61:197-208. [PubMed: 338630]

43. Willer CJ, Li Y, Abecasis GR. METAL: fast and efficient meta-analysis of genomewide association scans. Bioinformatics. 2010; 26:2190-1. [PubMed: 20616382]

44. van de Bunt M, et al. Insights into islet development and biology through characterization of a human iPSC-derived endocrine pancreas model. Islets. 2016; 8:83-95. [PubMed: 27246810]

45. Cong L, et al. Multiplex genome engineering using CRISPR/Cas systems. Science. 2013; 339:81923. [PubMed: 23287718]

46. Krentz NAJ, et al. Phosphorylation of NEUROG3 Links Endocrine Differentiation to the Cell Cycle in Pancreatic Progenitors. Dev Cell. 2017; 41:129-142.e6. [PubMed: 28441528]

47. Perez-Alcantara M, et al. Patterns of differential gene expression in a cellular model of human islet development, and relationship to type 2 diabetes predisposition. Diabetologia. 2018; 61:16141622. [PubMed: 29675560] 
48. Harries LW, Hattersley AT, Ellard S. Messenger RNA transcripts of the hepatocyte nuclear factor-1alpha gene containing premature termination codons are subject to nonsense-mediated decay. Diabetes. 2004; 53:500-4. [PubMed: 14747304]

49. Merriman C, Li H, Li H, Fu D. Highly specific monoclonal antibodies for allosteric inhibition and immunodetection of the human pancreatic zinc transporter ZnT8. J Biol Chem. 2018; 293:1620616216. [PubMed: 30181214]

50. Ravassard P, et al. A genetically engineered human pancreatic $\beta$ cell line exhibiting glucoseinducible insulin secretion. J Clin Invest. 2011; 121:3589-97. [PubMed: 21865645]

51. Thomsen SK, et al. Systematic Functional Characterization of Candidate Causal Genes for Type 2 Diabetes Risk Variants. Diabetes. 2016; 65:3805-3811. [PubMed: 27554474]

52. Chandra V, et al. RFX6 regulates insulin secretion by modulating $\mathrm{Ca} 2+$ homeostasis in human $\beta$ cells. Cell Rep. 2014; 9:2206-18. [PubMed: 25497100]

53. Ramracheya R, et al. Membrane potential-dependent inactivation of voltage-gated ion channels in alpha-cells inhibits glucagon secretion from human islets. Diabetes. 2010; 59:2198-208. [PubMed: 20547976]

54. Ottosson-Laakso E, et al. Glucose-Induced Changes in Gene Expression in Human Pancreatic Islets: Causes or Consequences of Chronic Hyperglycemia. Diabetes. 2017; 66:3013-3028. [PubMed: 28882899]

55. Fadista J, et al. Global genomic and transcriptomic analysis of human pancreatic islets reveals novel genes influencing glucose metabolism. Proc Natl Acad Sci U S A. 2014; 111:13924-9. [PubMed: 25201977]

56. Rosengren $\mathrm{AH}$, et al. Overexpression of alpha2A-adrenergic receptors contributes to type 2 diabetes. Science. 2010; 327:217-20. [PubMed: 19965390]

57. Taneera J, et al. Identification of novel genes for glucose metabolism based upon expression pattern in human islets and effect on insulin secretion and glycemia. Hum Mol Genet. 2015; 24:1945-55. [PubMed: 25489054]

58 . Bonetti S, et al. Variants of GCKR affect both $\beta$-cell and kidney function in patients with newly diagnosed type 2 diabetes: the Verona newly diagnosed type 2 diabetes study 2 . Diabetes Care. 2011; 34:1205-10. [PubMed: 21411509]

59. Li D, et al. Imaging dynamic insulin release using a fluorescent zinc indicator for monitoring induced exocytotic release (ZIMIR). Proc Natl Acad Sci U S A. 2011; 108:21063-8. [PubMed: 22160693]

60. Robinson MD, McCarthy DJ, Smyth GK. edgeR: a Bioconductor package for differential expression analysis of digital gene expression data. Bioinformatics. 2010; 26:139-140. [PubMed: 19910308] 
a

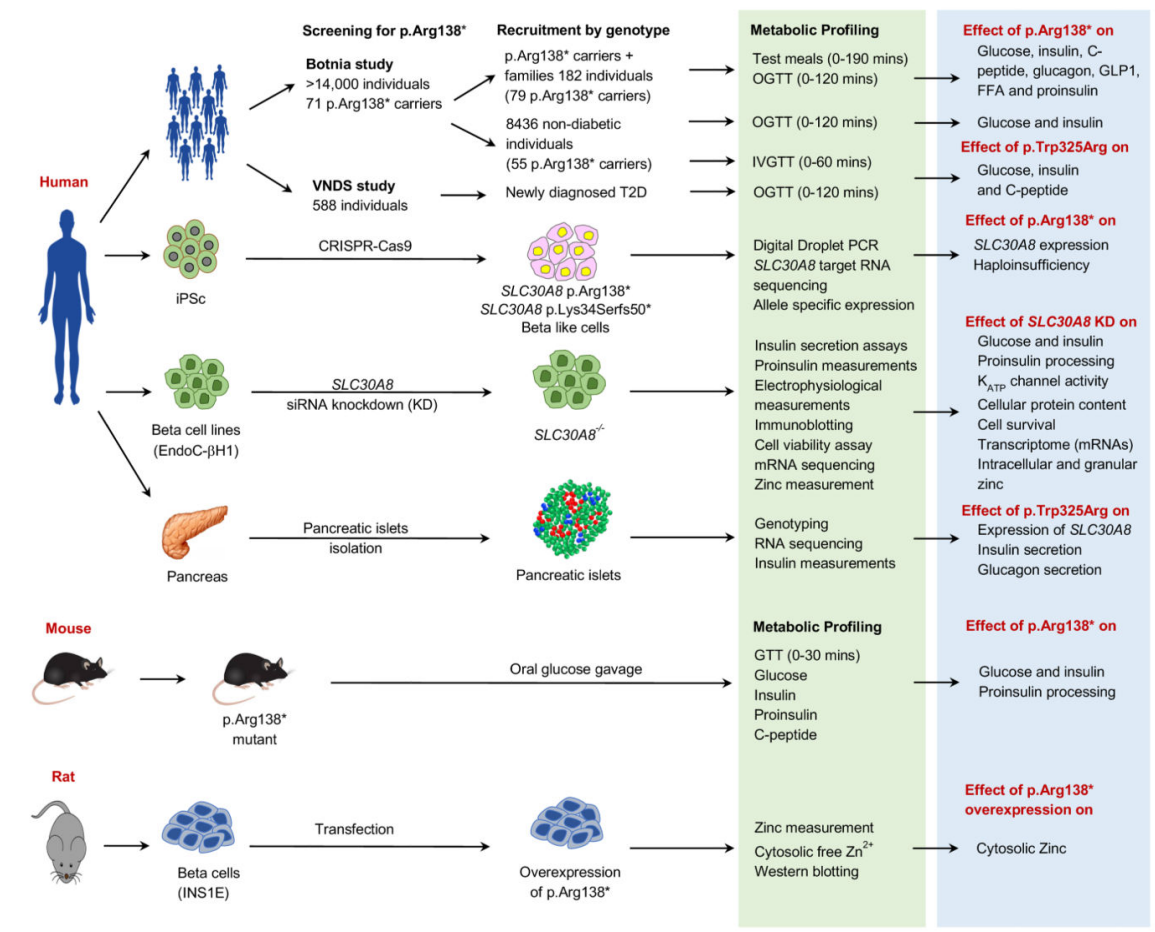

b

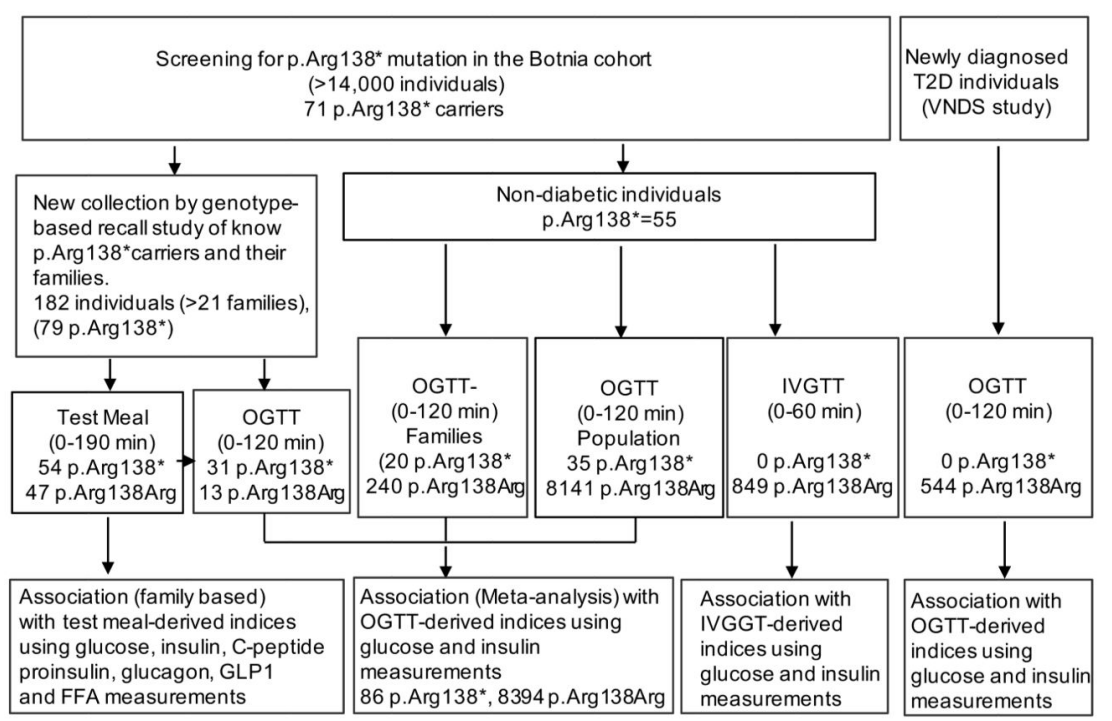

Fig. 1. A flow-chart describing the study design.

OGTT; oral glucose tolerance test, IVGTT; intravenous glucose tolerance test, GTT; glucose tolerance test

a, The study design including various model systems (left panels), methods (middle panels) and the purpose of these experiments (right panels).

b, Detailed description of the human in vivo studies, including a genotype-based recall study for p.Arg 138* carriers and their relatives for metabolic studies. 
a

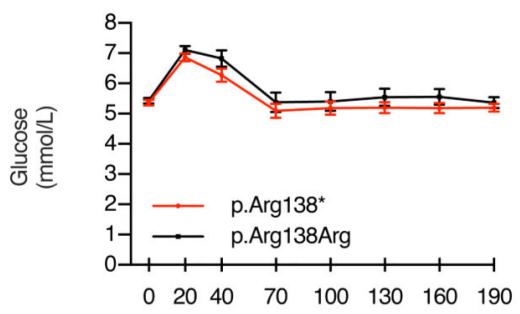

b

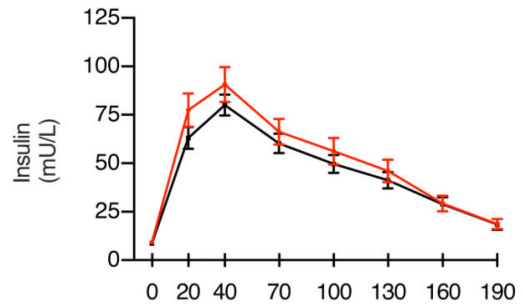

c

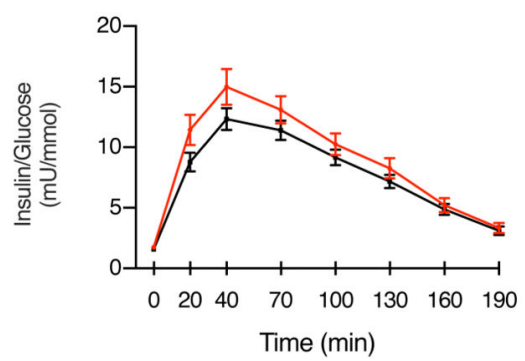

d
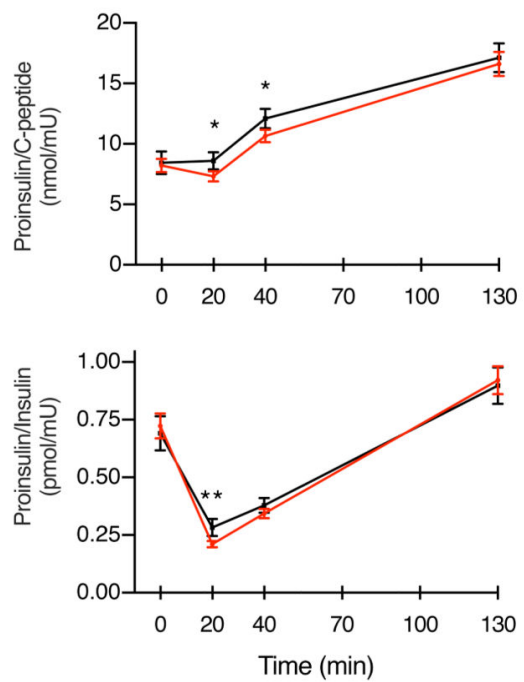
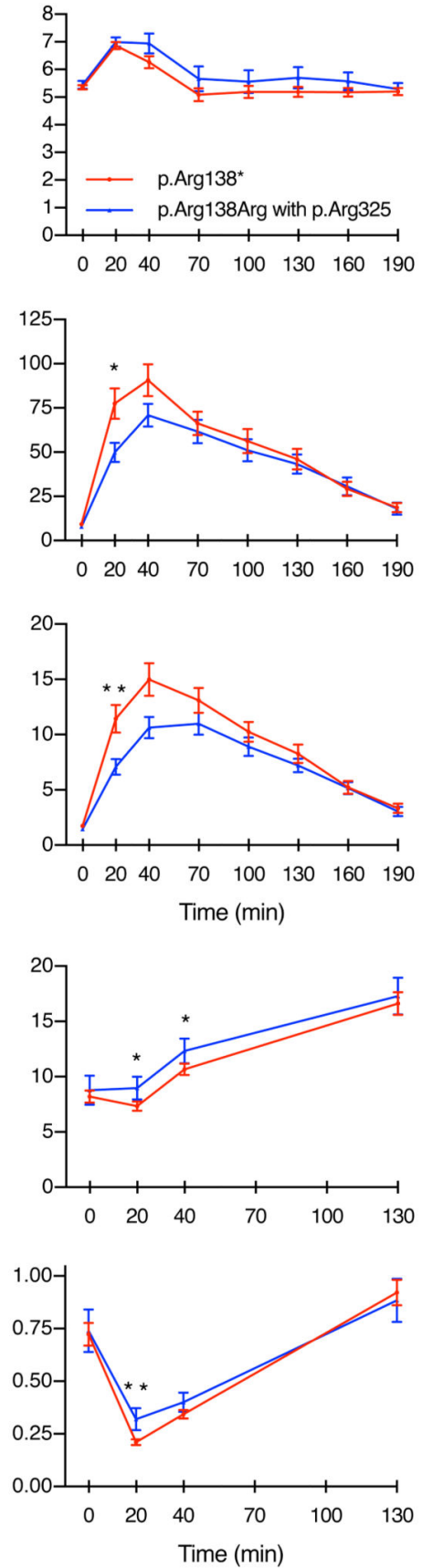
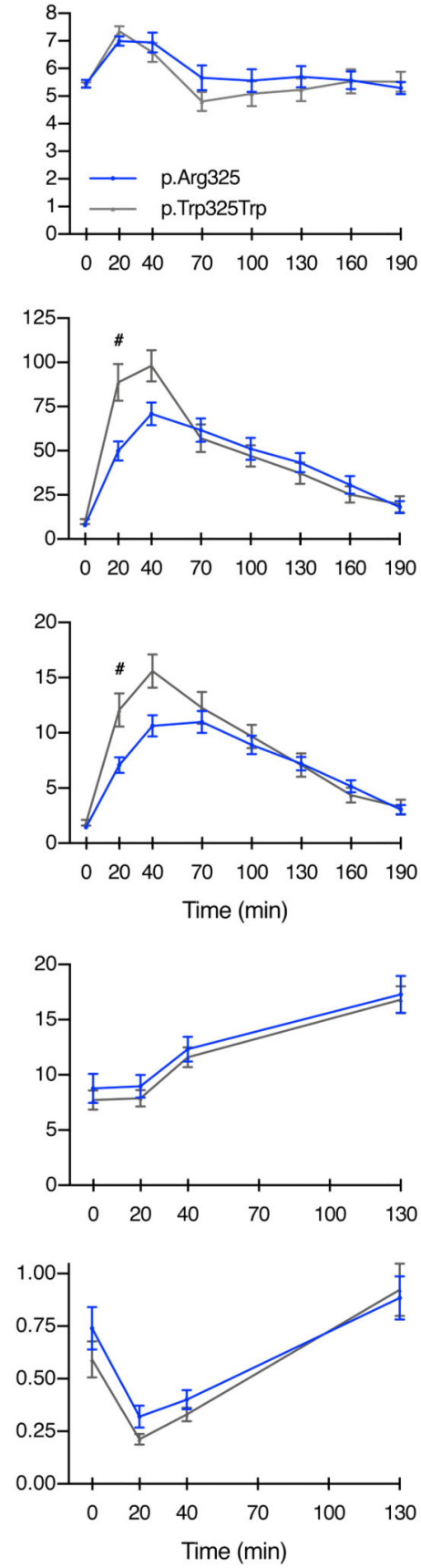

Fig. 2. SLC30A8-p.Arg138* enhances insulin secretion and proinsulin processing during test meal.

Association of $S L C 30 A 8$ p.Arg138* and p.Trp325Arg variants with a, plasma glucose b, serum insulin c, insulin/glucose ratio d, proinsulin/C-peptide ratio and e, proinsulin/insulin ratio during test meal. Left panel: Carriers (red, n=53-54) vs. non-carriers (black, n=37-47) of p.Arg138*. Middle panel: Carriers of p.Arg138* (red, n=53-54) vs Arg138Arg having the common risk variant p.Arg325 (blue, $\mathrm{n}=25-31$ ). Right panel: Carriers of p.Trp325Trp (grey, $\mathrm{n}=12-16$ ) vs. p.Arg325 (blue, $\mathrm{n}=31$ ).). Exact numbers used for genetic association analysis 
are available in the Source Data files. Data are Mean \pm SEM. A star $(* \mathrm{p}<0.05, * * \mathrm{p}<0.01)$ indicates significance in family based association (using QTDT ${ }^{35}$ ) after 100,000

permutations, adjusted for age, sex and BMI for left panel and age, sex, BMI and genotype of p.Trp325Arg for middle panel. A hash sign (\# p < 0.05) indicates significance in familybased QFAM (as implemented in PLINK ${ }^{36}$ ) test using 100,000 permutations (see Methods). 
a

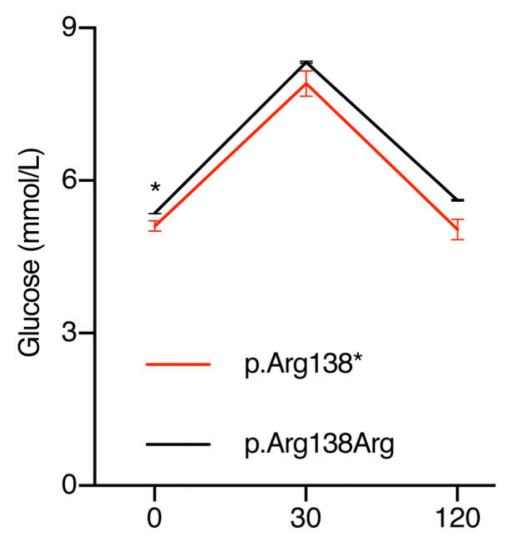

b
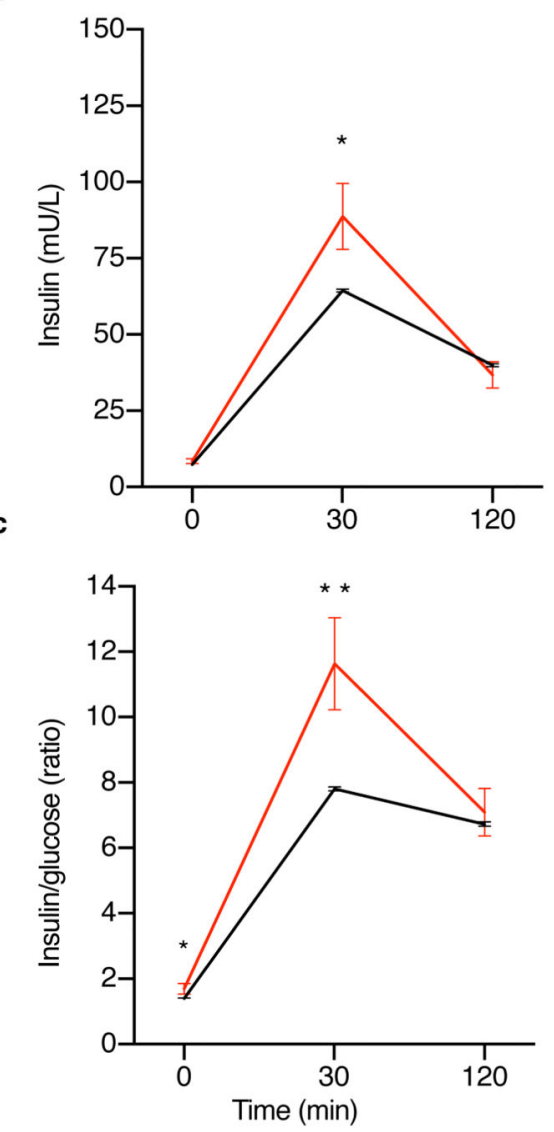
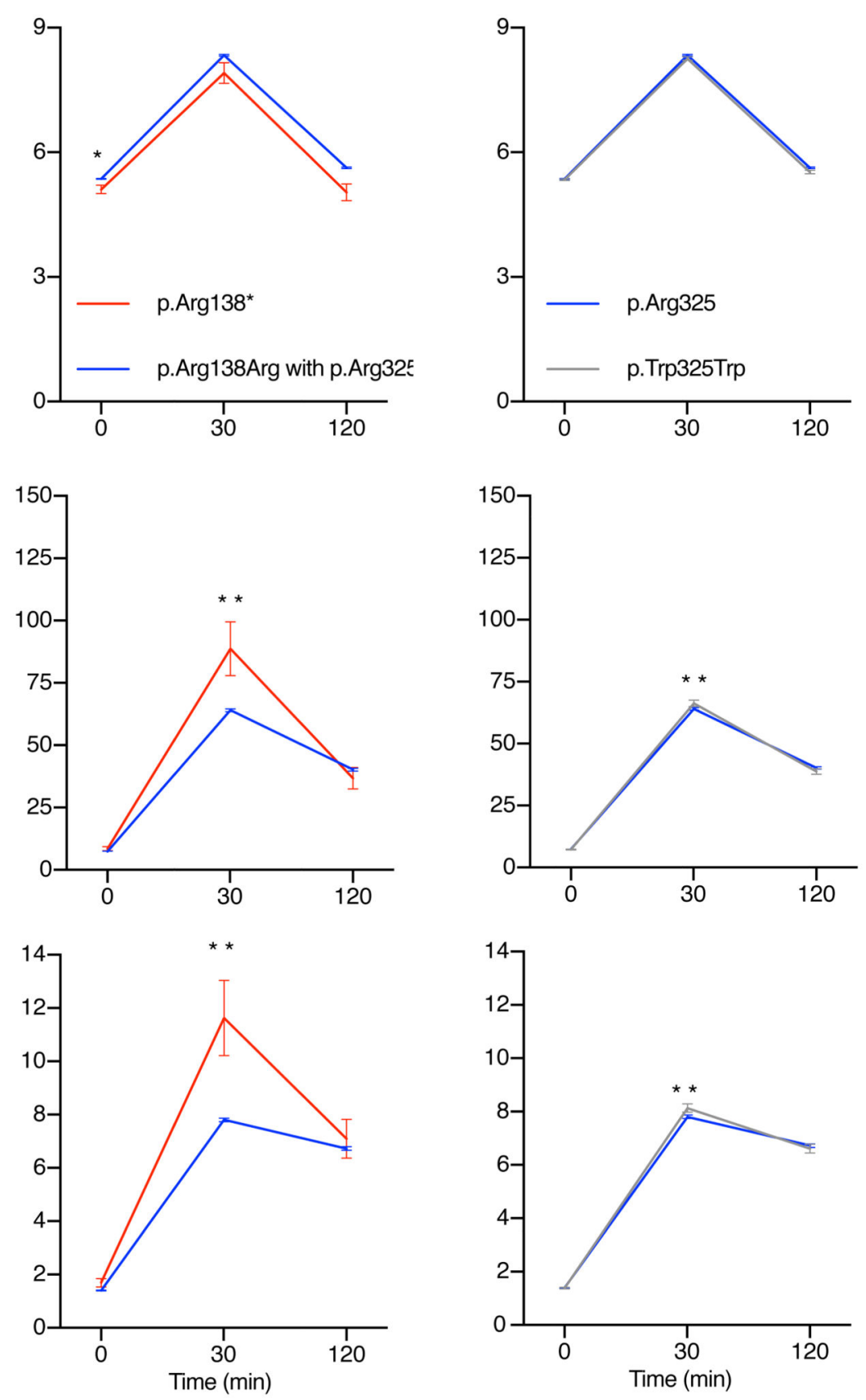

Fig. 3. SLC30A8 p.Arg138* and p.Trp325 enhance insulin secretion during OGTT. Association of SLC30A8 p.Arg 138* and p.Trp325Arg with a, plasma glucose b, serum insulin $\mathbf{c}$, insulin/glucose ratio during an oral glucose tolerance test (OGTT). Left panel: Carriers (red, $\mathrm{n}=34-35$ ) vs. non-carriers (black, $\mathrm{n}=7,954-8,141$ ) of $\mathrm{p}$.Arg138*. Middle panel: Carriers of p.Arg 138* (red, n=34-35) vs. p.Arg 138Arg having the common risk variant p.Arg325 (blue, $\mathrm{n}=6,728-6,893$ ). Right panel: Carriers of p.Trp325Trp (grey $\mathrm{n}=1,226-1,248$ ) vs. p.Arg325 (blue, $n=6,728-6,893$ ). Exact numbers used for genetic association analysis are available in Supplementary Table 5. Data are shown as Mean \pm SEM. A star $(*)$ indicates 
significance $(* \mathrm{p}<0.05, * * \mathrm{p}<0.01)$ for additive effects, calculated using mixed model adjusting for genetic relationship, age, sex, BMI and additionally genotype of p.Trp325Arg only for middle panel as implemented in GCTA ${ }^{37}$ (see Methods). 


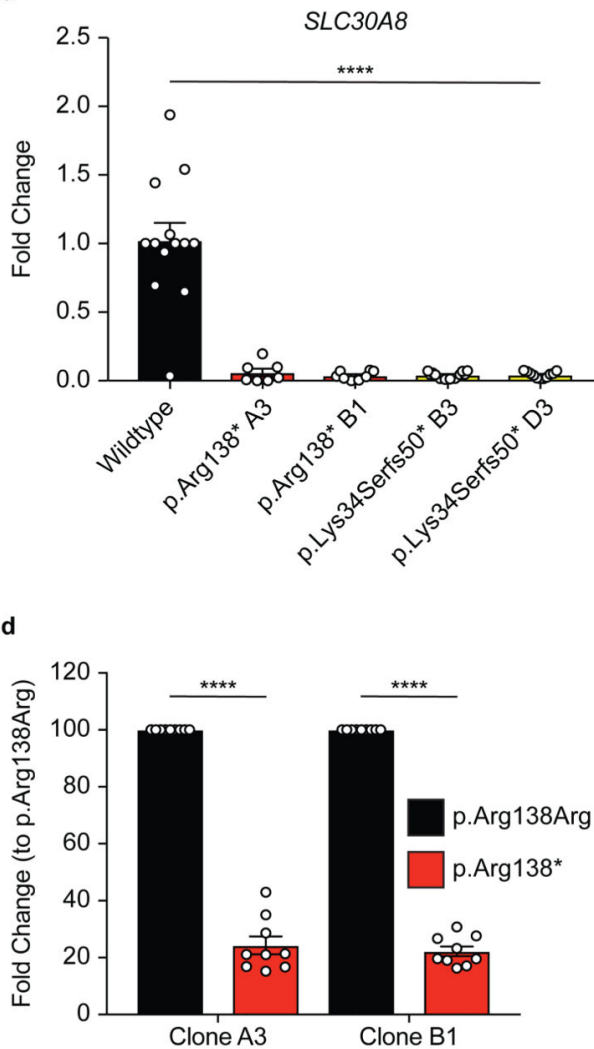

f

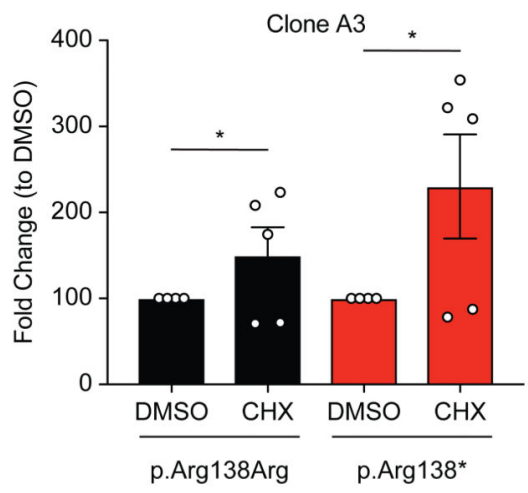

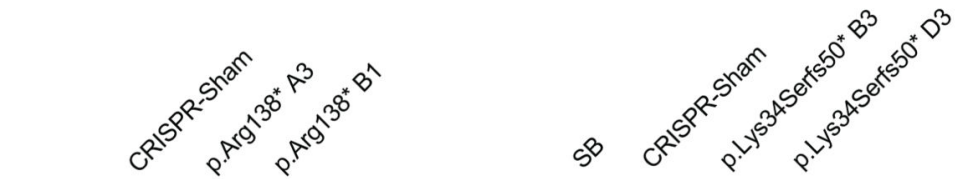

$34 \mathrm{kDa}-$

ZnT8

$34 \mathrm{kDa}-$

ZnT8

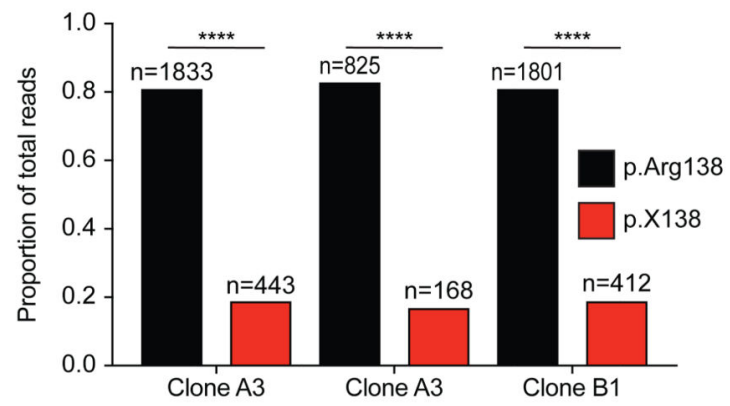

g

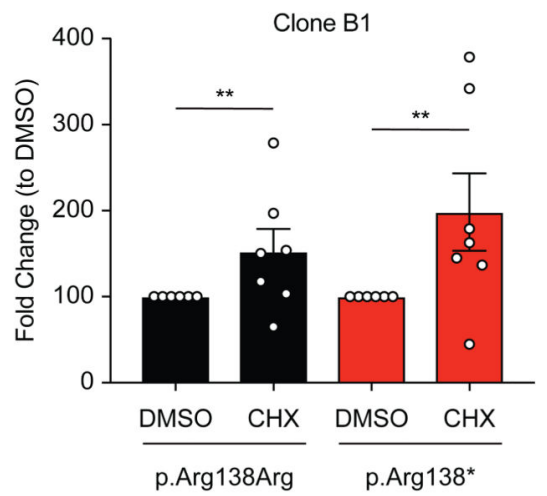

Fig. 4. Beta like cells derived from SLC30A8-p.Arg138* iPSCs display haploinsufficiency of SLC30A8.

a-c, Characterization of $S L C 30 A 8$ expression at the (a) mRNA and (b-c) protein level in cells heterozygous for SLC30A8-p.Arg138* or homozygous for SLC3OA8-

p.Lys34Serfs50*. Gene expression data normalized to $T B P$ and expressed as fold change relative to p.Arg138Arg control ( $n=7-13$ wells from three differentiations). d-e, Allelespecific expression (ASE) of p.Arg138Arg (black bar) and p.Arg138* (red bar) in clone A3 or clone B1 derived cells using (d) Digital Droplet PCR based probes also validated by (e) 
target $S L C 30 A 8$ mRNA sequencing of p.Arg $138^{*}$ clones (n=number of unique sequencing reads for each allele). f-g, Allele-specific expression of p.Arg138Arg (black bar) and p.Arg138* (red bar) in f, clone A3 and $\mathbf{g}$, clone B1 derived cells treated with DMSO (Dimethyl sulfoxide) or cycloheximide (CHX) for four hours. ASE data (Mean \pm SEM) determined by Digital Droplet PCR were presented as fold change relative to p.Arg138Arg transcript (d, $n=9$ wells from three differentiations) or to DMSO control (f-g, $n=4-7$ wells from two differentiation). Blots have been cropped and corresponding full blots are available in the Source Data files. *p<0.05, **p $<0.01, * * * \mathrm{p}<0.0001$ one-way ANOVA Holm-Sidak's multiple comparison test (a) or one-sample t-test (d, f and $\mathbf{g}$ ) or binominal test (one-tailed) considering 0.5 as expected probability (e). 

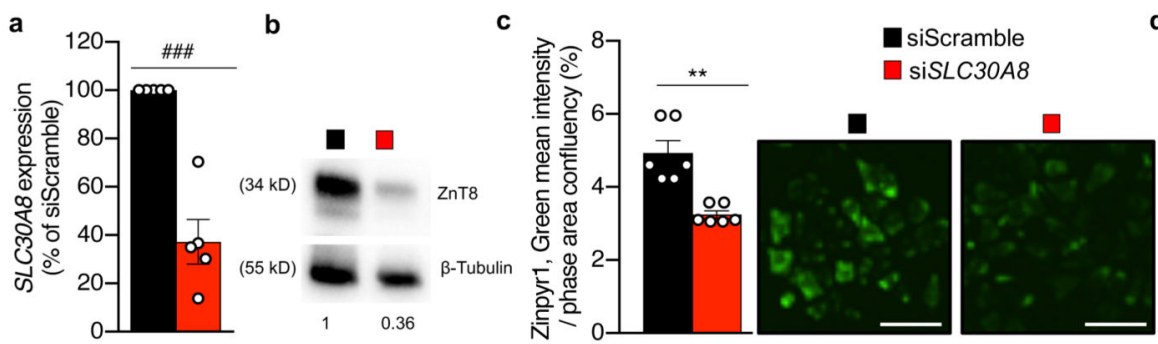

d

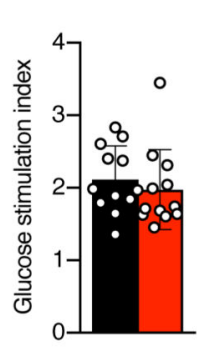

f

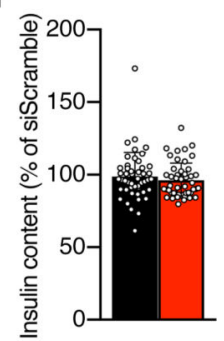

g

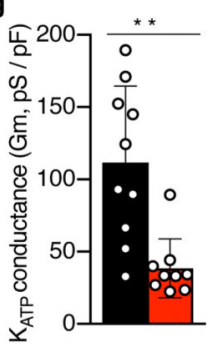

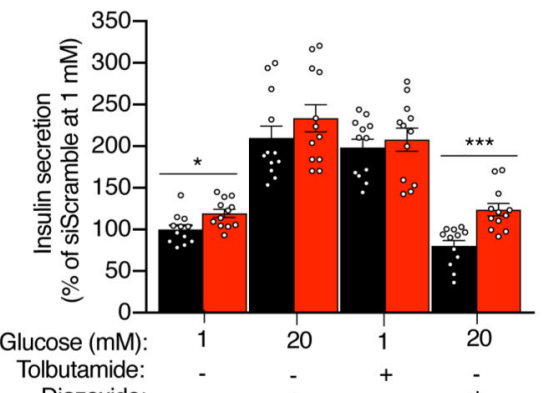
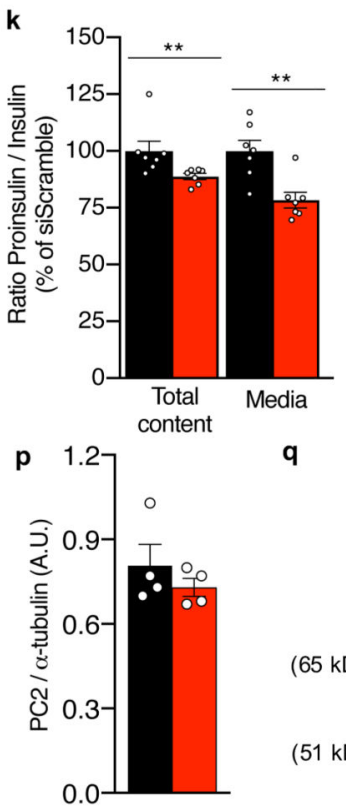
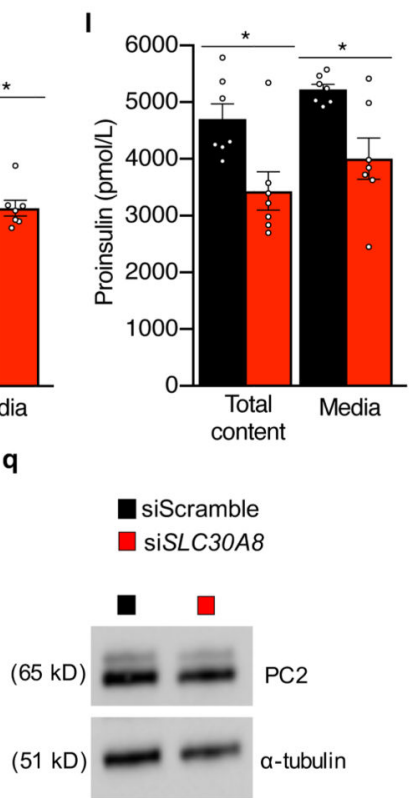

h
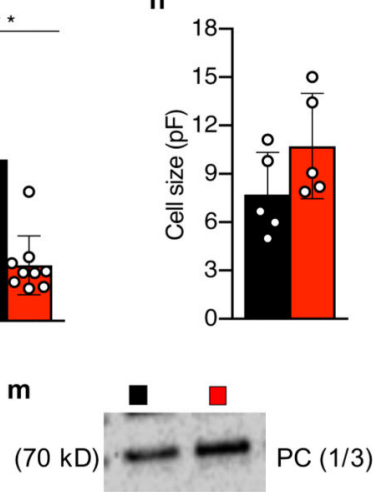

(51 kD)

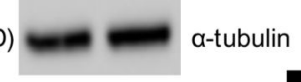

$(50 \mathrm{kD})$

(42 kD)

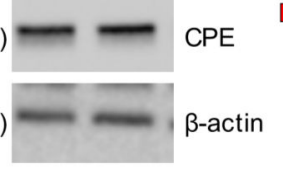

siScramble siSLC30A8<smiles>[AlH2]</smiles>

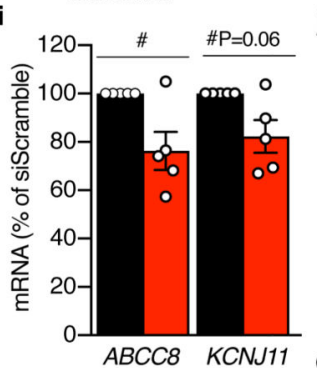
j

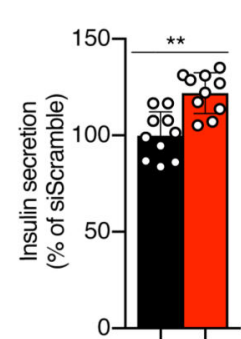
$\mathrm{KCL}(\mathrm{mM}): 50 \quad 50$

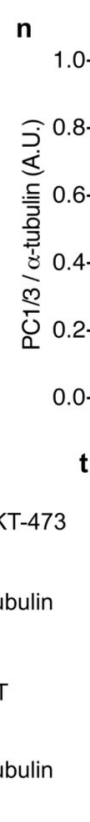
(1)

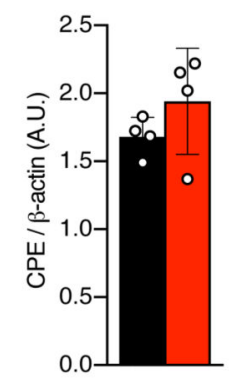

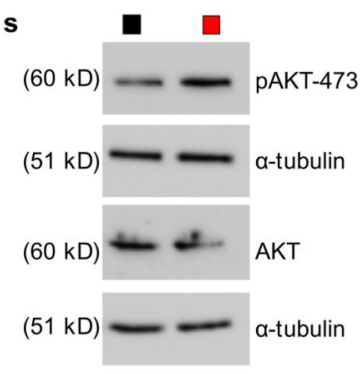

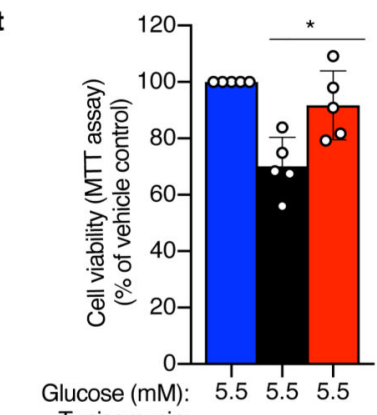

Fig. 5. SLC30A8 knockdown (KD) leads to enhanced insulin secretion, proinsulin processing and cell viability in the human pancreatic EndoC- $\beta$ h1 cells.

Black bar graph; control cells (siScramble); Red bar graph: SLC30A8 knock down cells (siSLC3OA8). a-b, Effect of siRNA mediated knock down (KD) on SLC30A8 mRNAs $(n=5)$ and protein. Intensities of the signal normalized to control condition are reported below the blot. c, Measurement of intracellular zinc using zinc-specific fluorescent dye Zinpyr-1 ( $\mathrm{n}=6$, scale bars=50 $\mu \mathrm{m})$. $\mathbf{d}-\mathbf{j}$, Effect of KD on $(\mathbf{d})$ insulin secretion (normalized to total insulin content and average siScramble basal secretion) stimulated by glucose and $\mathrm{K}_{\mathrm{ATP}}$ channel regulators (as labelled, and $\mathrm{n}=12$ ), (e) glucose stimulation index induced by 
20 mmol. $\mathrm{L}^{-1}$ glucose stimulation $(\mathrm{n}=12)$, and $(\mathbf{f})$ insulin content $(\mathrm{n}=48)$. $\mathbf{g}$, Effect of siSLC30A8 KD on $\mathrm{K}_{\mathrm{ATP}}$ channel conductance (Gm, siScramble $\mathrm{n}=10$ cells, siSLC30A8 $\mathrm{n}=9$ cells), (h) cell size $(n=5)$, (i) expression of $K_{\text {ATP }}$ channel subunits $(n=5)$, and (j) on insulin secretion stimulated by KCL and high glucose $(n=10)$. $\mathbf{k - l}$, Effect of KD on proinsulin processing estimated by $(\mathbf{k})$ proinsulin/insulin ratio $(n=7)$ and (l) proinsulin concentration $(\mathrm{n}=7)$. $\mathbf{m}-\mathbf{q}$, Effect of KD on protein expression of proinsulin processing enzymes PC1/3, CPE (immunoblot- $\mathbf{m}$ and densitometry- $\mathbf{n}, \mathbf{o} ; \mathbf{n}, \mathbf{n}=5$ and $\mathbf{o}, \mathbf{n}=4$ ) and PC2 (densitometry- $\mathbf{p}$, $\mathrm{n}=4$ and immunoblot- $\mathbf{q})$. r-t, Effect of KD on basal (5.5 mM glucose) AKT phosphorylation (densitometry- r, n=5 and immunoblot- s; phospho-AKT-Ser473, total AKT) and cell viability under ER stress (t, MTT assay, $n=5)$. Blots have been cropped and corresponding full blots are available in the Source Data files. Data are Mean \pm SEM. $* p<0.05, * * p<0.01$, $* * * \mathrm{p}<0.001$ using Mann-Whitney test; \#p<0.05 one-sample t-test; $\mathbf{d}$ (Bonferroni multiple correction for 4 conditions). 

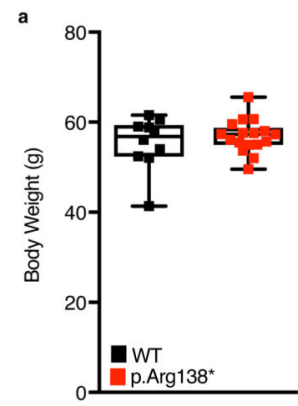

d

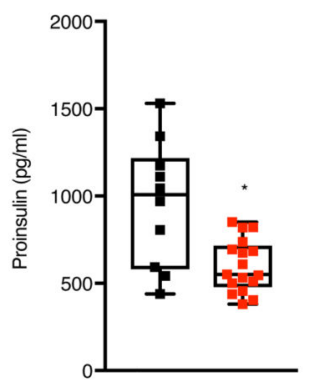

g
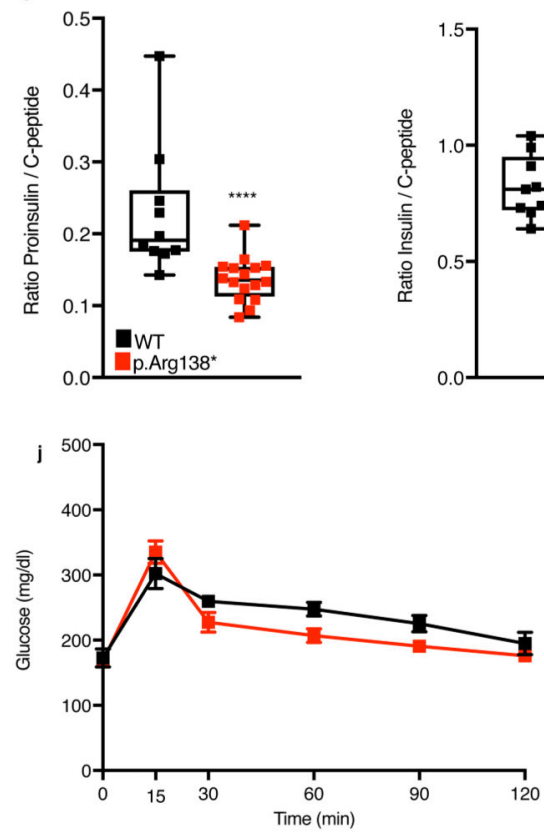
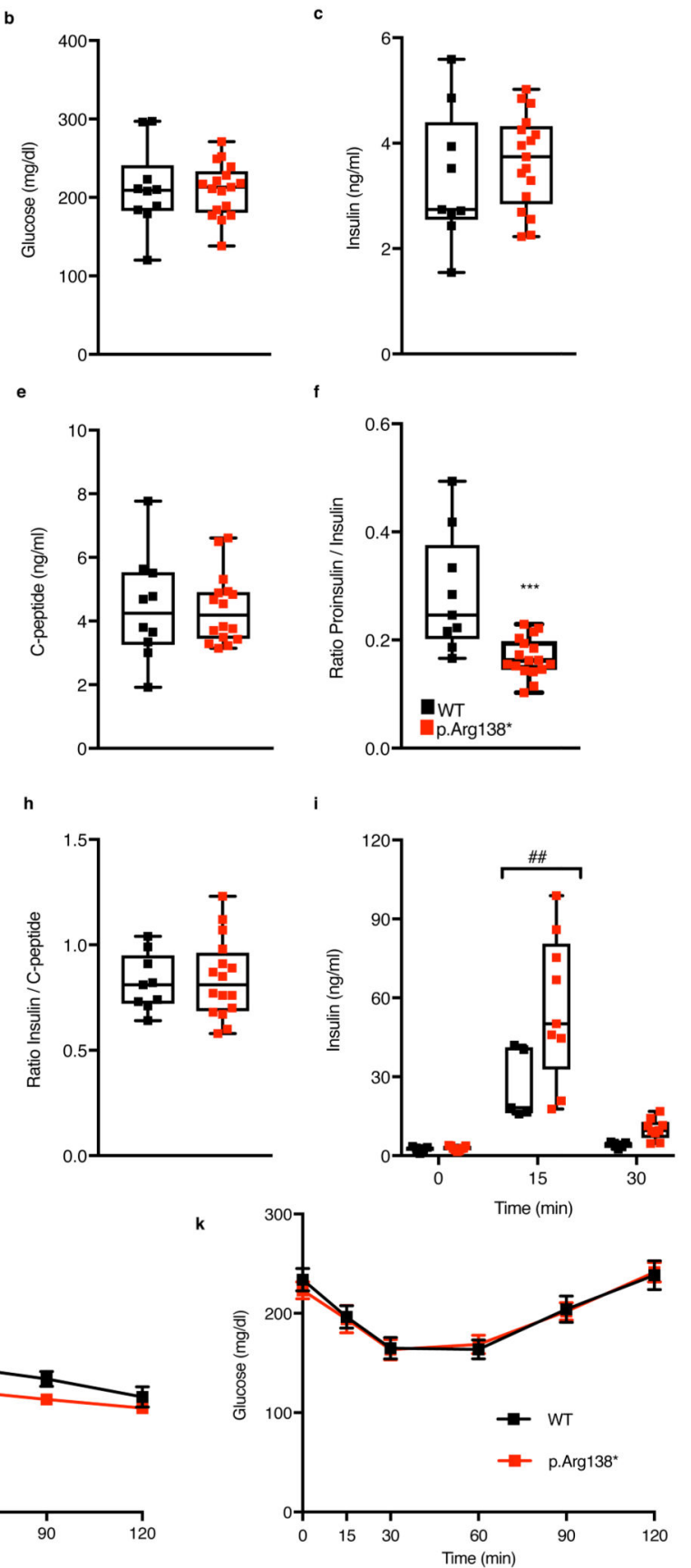

Fig. 6. Male Slc30a8 p.Arg138* mice on high-fat diet show enhanced insulin secretion and proinsulin processing.

Black boxes; wild type mice (WT), Red boxes; Slc30a8 p.Arg138* mice. a, Body weight $\left(\mathrm{n}=10 / 17 \mathrm{WT} / \mathrm{p} . \operatorname{Arg} 138^{*}\right)$, circulating b, glucose (n=10/17 WT/p.Arg138*) c, insulin $\left(\mathrm{n}=9 / 17 \mathrm{WT} / \mathrm{p} . \operatorname{Arg} 138^{*}\right) \mathbf{d}$, proinsulin $\left({ }^{*} \mathrm{p}=0.011, \mathrm{n}=10 / 17 \mathrm{WT} / \mathrm{p}\right.$.Arg138*) e, C-peptide $\left(\mathrm{n}=10 / 16 \mathrm{WT} / \mathrm{p} . \operatorname{Arg} 138^{*}\right) \mathbf{f}$, proinsulin/insulin ratio $\left(\mathrm{n}=9 / 17 \mathrm{WT} / \mathrm{p} . \operatorname{Arg} 138^{*}\right) \mathbf{g}$, proinsulin/C-peptide ratio ( $\mathrm{n}=10 / 16 \mathrm{WT} / \mathrm{p}$.Arg138*) and $\mathbf{h}$, insulin/C-peptide ratio $(\mathrm{n}=9 / 16$ WT/p.Arg138*) in fasted WT and p.Arg138* mice after 20 weeks on HFD. i, Insulin 
response to oral glucose $(2 \mathrm{~g} / \mathrm{kg})$ exposure ( $\left.\mathrm{n}=5 / 9 \mathrm{WT} / \mathrm{p} . \operatorname{Arg} 138^{*}\right)$ after 30 weeks on HFD. Blood glucose levels over time after $\mathbf{j}$, oral glucose $(2 \mathrm{~g} / \mathrm{kg}$ ) exposure ( $\mathrm{n}=5 / 11 \mathrm{WT} /$ p.Arg 138*) after 29 weeks on HFD and $\mathbf{k}$, interperitoneal injection of insulin (1.75 U/kg) after 28 weeks on HFD ( $\mathrm{n}=11 / 13 \mathrm{WT} / \mathrm{p}$. Arg 138*). Boxplot depicts the interquartile range, median and minimum/maximum values. ${ }^{*} \mathrm{p}<0.05$, ${ }^{* * *} \mathrm{p}<0.001,{ }^{* * * *} \mathrm{p}<0.0001$ using Mann Whitney test; ${ }^{\#} \mathrm{p}<0.05,{ }^{\#} \mathrm{p}<0.01$ using two-way Anova (repeated measurements) and Sidak's multiple comparison test. 
a

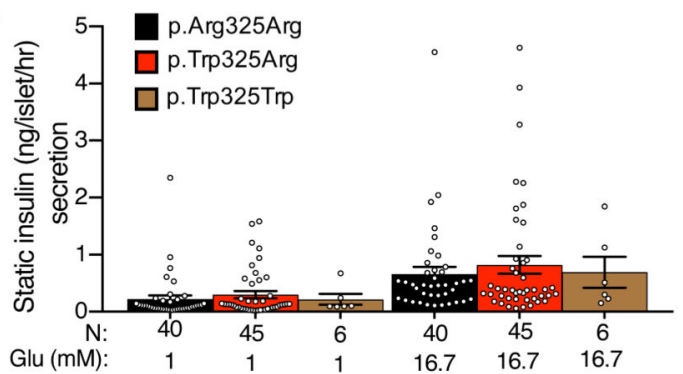

c
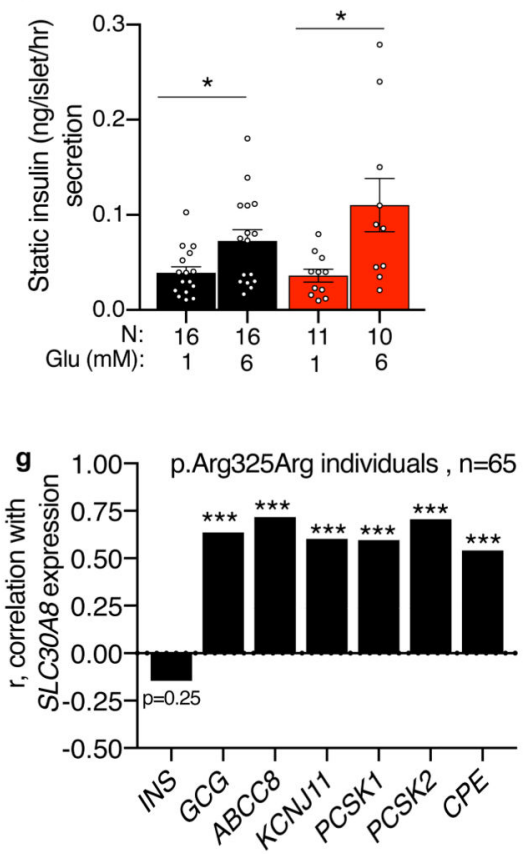

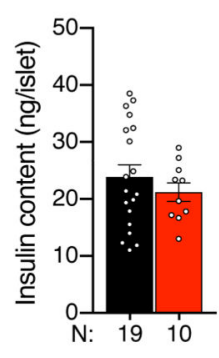

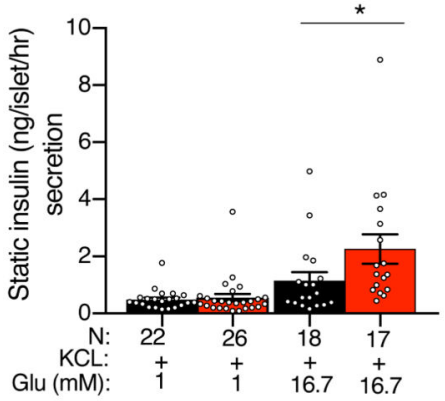

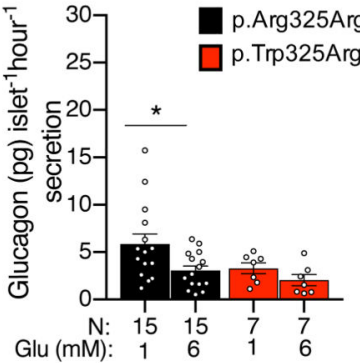

f
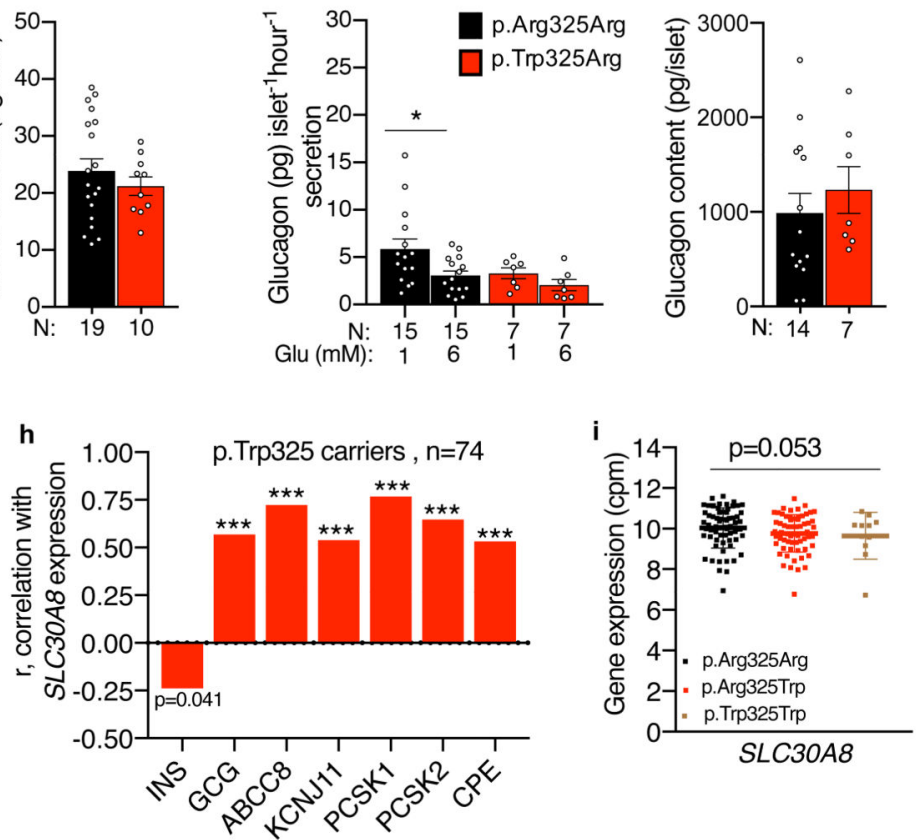

j

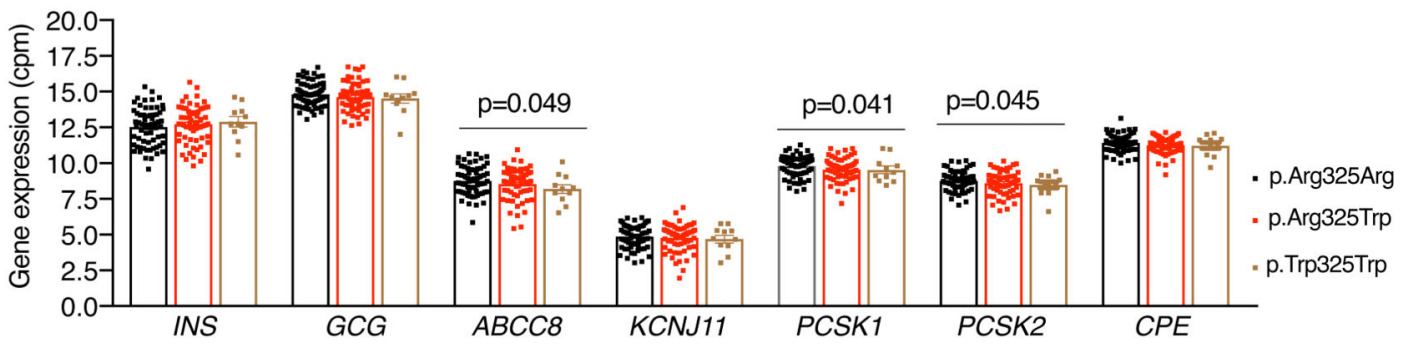

Fig. 7. SLC30A8- p.Trp325 leads to enhanced insulin secretion in human islets.

Experiments have been performed in two different centers: LUDC (a, b, g, h, i and j) and Oxford (c, d, e and f). a, Effect of p.Trp325Arg genotype on static insulin secretion in presence of low and high glucose stimulatory conditions. $\mathbf{b}$, Effect of p.Trp325Arg genotype on static insulin secretion in presence of low or high glucose and KCL. c-d, Effect of p.Trp325Arg genotype on static insulin secretion in (c) sub-maximal stimulatory conditions (6mM glucose) and their (d) insulin contents. e, Static glucagon response to glucose and $\mathbf{f}$, glucagon content at basal glucose. $\mathbf{g}-\mathbf{h}$, Correlation of $S L C 30 A 8$ expression with candidate 
genes of $I N S, G C G$, proinsulin processing genes and $\mathrm{K}_{\mathrm{ATP}}$ channel subunits genes among (g) p.Arg325Arg individuals and (h) p.Trp325 carriers and effect of p.Trp325Arg genotype (p.Arg325Arg=65, p.Trp325Arg=63 and p.Trp325Trp=11) on expression $(\mathrm{cpm}=\log 2$ of counts per million) of (i) $S L C 3 O A 8$ and (j) other candidate genes. Data are Mean \pm SEM, Glu; glucose, $\mathrm{N}$; number of experiments. ${ }^{*} \mathrm{p}<0.05,{ }^{* * *} \mathrm{p}<0.0001$ using Mann-Whitney test $(\mathbf{b}, \mathbf{c}, \mathbf{d}, \mathbf{e}$ and $\mathbf{f}$ ) or Spearman correlation coefficient ( $\mathrm{r}$ ) with two-tailed $\mathrm{p}$ values ( $\mathbf{g}$ and $\mathbf{h}$ ). Three genotype comparison $(\mathbf{a}, \mathbf{i}$ and $\mathbf{j})$ by linear regression using additive effect adjusting for age, sex and islet purity as implemented in LLINK $^{36}$ (see Methods). 